TRANSACTIONS OF THE

AMERICAN MATHEMATICAL SOCIETY

Volume 362, Number 9, September 2010, Pages 4871-4900

S 0002-9947(10)05038-5

Article electronically published on April 8, 2010

\title{
SLICELY COUNTABLY DETERMINED BANACH SPACES
}

\author{
ANTONIO AVILÉS, VLADIMIR KADETS, MIGUEL MARTÍN, JAVIER MERÍ, \\ AND VARVARA SHEPELSKA
}

\begin{abstract}
We introduce the class of slicely countably determined Banach spaces which contains in particular all spaces with the Radon-Nikodým property and all spaces without copies of $\ell_{1}$. We present many examples and several properties of this class. We give some applications to Banach spaces with the Daugavet and the alternative Daugavet properties, lush spaces and Banach spaces with numerical index 1 . In particular, we show that the dual of a real infinite-dimensional Banach space with the alternative Daugavet property contains $\ell_{1}$ and that operators which do not fix copies of $\ell_{1}$ on a space with the alternative Daugavet property satisfy the alternative Daugavet equation.
\end{abstract}

\section{INTRODUCTION}

The aim of this paper is to introduce the class of slicely countably determined Banach spaces, give many examples and several properties of this class and, finally, use this concept to give some applications to Banach spaces with the Daugavet property and to Banach spaces with numerical index 1. Let us introduce the needed notation and definitions.

Given a Banach space over $\mathbb{K}(\mathbb{K}=\mathbb{R}$ or $\mathbb{K}=\mathbb{C})$, we write $S_{X}$ for its unit sphere and $B_{X}$ for its closed unit ball. The dual space of $X$ is denoted by $X^{*}$, and $L(X)$ is the Banach algebra of all bounded linear operators from $X$ to $X$. The space $X$ has the Daugavet property [19] if every rank-one operator $T \in L(X)$ satisfies

$$
\|\mathrm{Id}+T\|=1+\|T\| .
$$

In this case, all operators on $X$ which do not fix copies of $\ell_{1}$ (in particular, weakly compact operators) also satisfy (DE) 29]. If every rank-one operator $T \in L(X)$ satisfies the norm equality

$$
\max _{\theta \in \mathbb{T}}\|\operatorname{Id}+\theta T\|=1+\|T\|
$$

Received by the editors September 15, 2008 and, in revised form, March 2, 2009

2010 Mathematics Subject Classification. Primary 46B20; Secondary 46B03, 46B04, 46B22, $47 \mathrm{~A} 12$.

Key words and phrases. Numerical radius, numerical index, Daugavet equation, RadonNikodým property, Asplund spaces, containing of $\ell_{1}$, narrow operators.

The first-named author was supported by the Marie Curie Intra-European Fellowship MCEIFCT2006-038768 (EU) and the Spanish research project MTM2005-08379 (MEC and FEDER). The second-named author was supported by Junta de Andalucía and FEDER grant P06-FQM-01438. The third- and fourth-named authors were partially supported by the Spanish MEC and FEDER project no. MTM2006-04837 and Junta de Andalucía and FEDER grants FQM-185 and P06FQM-01438. The fifth-named author was partially supported by the N. I. Akhiezer Foundation. 
( $\mathbb{T}$ being the set of modulus one scalars), $X$ has the alternative Daugavet property 25. In this case, all weakly compact operators on $X$ also satisfy (aDE). A Banach space is said to have numerical index 1 [13] if every $T \in L(X)$ satisfies $v(T)=\|T\|$, where

$$
v(T)=\left\{\left|x^{*}(T x)\right|: x \in S_{X}, x^{*} \in S_{X^{*}}, x^{*}(x)=1\right\}
$$

is the numerical radius of the operator $T$. It is known [13] that

$$
v(T)=\|T\| \quad \Longleftrightarrow \quad T \text { satisfies (aDE). }
$$

Then, $X$ has numerical index 1 if and only if every $T \in L(X)$ satisfies (aDE). It follows from the above discussion that

$$
\text { Daugavet property } \Longrightarrow \text { Alternative Daugavet property } \Longleftarrow \text { Numerical index } 1 \text {. }
$$

None of the above implications reverses in general [25, Example 3.2]. For the first implication, it is even known that it is not reversible under any isomorphic property [25, Corollary 3.3]. On the other hand, it is known that the second implication reverses for Asplund spaces and for Banach spaces with the RadonNikodým property [23, Remark 6]. We refer the interested reader to [15, 16, 18, 24, and the already cited references for recent results, more information and background on these properties.

We will say that $X$ is slicely countably determined ( $S C D$ in short) if every bounded convex subset $A$ of $X$ is an $S C D$ set, i.e. there is a sequence $\left\{S_{n}: n \in \mathbb{N}\right\}$ of slices of $A$ such that $A \subseteq \overline{\operatorname{conv}}(B)$ whenever $B \subseteq A$ intersects all the $S_{n}$ 's. Here a slice of a convex set $A$ is the subset given by

$$
S\left(A, x^{*}, \varepsilon\right)=\left\{x \in A: \operatorname{Re} x^{*}(x)>\sup \operatorname{Re} x^{*}(A)-\varepsilon\right\}
$$

and $\overline{\operatorname{conv}}(\cdot)$ stands for the closed convex hull. This isomorphic property, which clearly implies separability, is sufficient to get numerical index 1 from the alternative Daugavet property, and it is weaker than both RNP and being Asplund (for separable spaces). Actually, this property is satisfied by both separable strongly regular spaces and separable Banach spaces which do not contain copies of $\ell_{1}$. This is the main motivation of the study of SCD spaces.

In section 2 we study SCD sets, giving examples and elementary properties. We show, for instance, that the sequence of slices can be replaced by a sequence of relatively weakly open sets or by a sequence of convex combinations of slices. In section 3 we study SCD spaces and show some stability properties. For instance, it is a three space property, so it is stable for finite sums, and it is stable for some infinite unconditional sums.

Since it is not easy to deal with Banach spaces with numerical index 1, there are in the literature several geometrical sufficient conditions (see [18), the weakest one being the so-called lushness. A Banach space $X$ is said to be lush [8] if for every $x, y \in S_{X}$ and every $\varepsilon>0$, there is a slice $S=S\left(B_{X}, x^{*}, \varepsilon\right)$ with $x^{*} \in S_{X^{*}}$ such that $x \in S$ and $\operatorname{dist}(y, \operatorname{aconv}(S))<\varepsilon$ (where $\operatorname{aconv}(A)$ denotes the absolutely convex hull of the set $A$ ). Lush spaces have numerical index 1 [8, Proposition 2.2], but it has been very recently shown that the converse result is not true [17. We refer to [7, 8, for background.

It is actually shown in section 4 that an SCD Banach space with the alternative Daugavet property is lush. This result allows us to show that $\ell_{1}$ embeds in the dual of every real infinite-dimensional Banach space with the alternative Daugavet property. This answers in the positive [18, Problem 18]. 
Section 5 is devoted to SCD-operators and hereditary-SCD-operators. A bounded linear operator $T: X \longrightarrow Y$ between two Banach spaces $X$ and $Y$ is said to be an $S C D$-operator if $T\left(B_{X}\right)$ is an SCD set, and $T$ is a hereditary-SCD-operator if every bounded convex subset of $T\left(B_{X}\right)$ is SCD. We show that SCD-operators on a Banach space with the alternative Daugavet property satisfy (aDE). Therefore, operators which do not fix copies of $\ell_{1}$ on a Banach space with the alternative Daugavet property satisfy (aDE). For a Banach space with the Daugavet property, it is shown that every SCD-operator is strong Daugavet (and so it satisfies (DE)) and every hereditary-SCD-operator is narrow.

Section 6 is devoted to the study of sets with a countable $\pi$-base of the weak topology. It is shown in section 2 that these sets are SCD, but it is not known whether the converse result is true. It is also shown in section 2 that separable sets without $\ell_{1}$ sequences have countable $\pi$-bases of the weak topology, and in this section we show that the same is true for $\mathrm{CPCP}$ sets and for bounded convex subsets of both $c_{0}\left(\ell_{1}\right)$ and $\ell_{1}\left(c_{0}\right)$. We also show some characterizations of SCD sets which remind us of the existence of countable $\pi$-bases of the weak topology. One of these characterizations allows us to show that the set of extreme points of the weak*-closure (in the bidual space) of an SCD set has a countable $\pi$-base of the weak ${ }^{*}$ topology, and so it is weak* separable. The set of extreme points of a convex set $B$ will be denoted by $\operatorname{ext}(B)$.

Finally, section 7 contains several open questions.

\section{Slicely COUntably Determined Sets}

Definition 2.1. Let $X$ be a Banach space and let $A$ be a convex bounded subset of $X$. A countable family $\left\{V_{n}: n \in \mathbb{N}\right\}$ of subsets of $A$ is called determining for $A$ if $A \subseteq \overline{\operatorname{conv}}(B)$ for every $B \subseteq A$ intersecting all the sets $V_{n}$. Equivalently, $\left\{V_{n}: n \in \mathbb{N}\right\}$ is determining for $A$ if for every sequence $\left\{v_{n}\right\}_{n \in \mathbb{N}}$ with $v_{n} \in V_{n}$ $(n \in \mathbb{N})$, one has $A \subseteq \overline{\operatorname{conv}}\left(\left\{v_{n}: n \in \mathbb{N}\right\}\right)$.

We give three easy observations which will be useful later on. The first one is a consequence of the Hahn-Banach theorem. The second and third ones are straightforward.

Proposition 2.2. Let $X$ be a Banach space and let $A$ be a convex bounded subset of $X$. A sequence $\left\{V_{n}: n \in \mathbb{N}\right\}$ of subsets of $A$ is determining if and only if every slice of $A$ contains one of the $V_{n}$.

Proof. The "if" part is evident: if $B \subseteq A$ intersects all the $V_{n}$, then it intersects all the slices of $A$, and then by the Hahn-Banach theorem $\overline{\operatorname{conv}}(B) \supseteq A$. Now the "only if" part. Assume that some slice $S$ of $A$ does not contain any of the $V_{n}$. Then $A \backslash S$ is a convex relatively closed subset of $A$ intersecting all the $V_{n}$. But $A \backslash S \neq A$, which means that $\left\{V_{n}: n \in \mathbb{N}\right\}$ is not determining.

Remark 2.3. Let $X$ be a Banach space and let $A$ be a convex bounded subset of $X$. Suppose that there is a sequence $\left\{a_{n}: n \in \mathbb{N}\right\}$ of points in $A$ such that $A \subseteq$ $\overline{\operatorname{conv}}\left(\left\{a_{n}: n \in \mathbb{N}\right\}\right)$ and that for every $n \in \mathbb{N}$, there is a sequence $\left\{V_{n, m}: m \in \mathbb{N}\right\}$ of subsets of $A$ such that $a_{n} \in \overline{\operatorname{conv}}(B)$ whenever $B \subseteq A$ intersects $V_{n, m}$ for every $m \in \mathbb{N}$. Then, the family $\left\{V_{n, m}: n, m \in \mathbb{N}\right\}$ is determining for $A$.

As an immediate consequence of the above result, we get the following. 
Remark 2.4. Let $X$ be a Banach space and let $A$ be a separable convex bounded subset of $X$. Suppose that for every $a \in A$ there is a sequence $\left\{V_{m}^{a}: m \in \mathbb{N}\right\}$ of subsets of $A$ such that $a \in \overline{\operatorname{conv}}(B)$ whenever $B \subseteq A$ intersects $V_{m}^{a}$ for every $m \in \mathbb{N}$. Then, taking a dense sequence $\left\{a_{n}: n \in \mathbb{N}\right\}$ in $A$, the family $\left\{V_{m}^{a_{n}}: n, m \in \mathbb{N}\right\}$ is determining for $A$.

We can now give the main definition of this section.

Definition 2.5. A convex bounded subset $A$ of a Banach space $X$ is said to be slicely countably determined (an $S C D$ set for short) if there is a determining sequence of slices of $A$.

Two remarks are pertinent.

Remark 2.6. It is clear from the definition that every SCD set is separable.

Remark 2.7. A convex bounded subset $A$ of a Banach space $X$ is SCD if and only if the closure of $A$ is an SCD set.

Proof. Let us show first that $\bar{A}$ is SCD when $A$ is SCD. Consider a determining sequence of slices $S_{n}=S\left(A, x_{n}^{*}, \varepsilon_{n}\right)(n \in \mathbb{N})$ for $A$, and let us prove that the slices $S_{n}^{\prime}=S\left(\bar{A}, x_{n}^{*}, \varepsilon_{n} / 2\right)(n \in \mathbb{N})$ form a determining sequence for the closure of $A$. Consider an arbitrary slice $S=S\left(\bar{A}, x^{*}, \varepsilon\right)$ of $\bar{A}$. Then, $S\left(\bar{A}, x^{*}, \varepsilon / 2\right) \cap A=$ $S\left(A, x^{*}, \varepsilon / 2\right)$ is a slice of $A$, so there is $n \in \mathbb{N}$ such that $S\left(A, x^{*}, \varepsilon / 2\right) \supseteq S_{n}$ by Proposition 2.2. Therefore, $S$ contains the closure of $S_{n}$, which in turn contains $S_{n}^{\prime}$, and again Proposition 2.2 gives us the fact that $\left\{S_{n}^{\prime}\right\}$ is determining for $\bar{A}$.

For the converse implication, we consider a determining sequence $\left\{S\left(\bar{A}, x_{n}^{*}, \varepsilon_{n}\right)\right.$ : $n \in \mathbb{N}\}$ for $\bar{A}$, and it is straightforward to show that $\left\{S\left(A, x_{n}^{*}, \varepsilon_{n}\right): n \in \mathbb{N}\right\}$ is determining for $A$.

Our first goal is to present the basic examples related to Definition 2.5: RadonNikodým and Asplund sets are SCD, whereas the unit ball of a Banach space with the Daugavet property is not.

We start with subsets having sufficiently many denting points. Let $X$ be a Banach space and let $A$ be a closed convex bounded subset of $X$. A point of $A$ is said to be a denting point if it belongs to slices of $A$ of arbitrarily small diameter. We write $\operatorname{dent}(A)$ to denote the set of denting points of $A$. We say that $A$ is dentable (in the sense of Ghoussoub-Godefroy-Maurey-Schachermayer [14, §III]) if $A=\overline{\operatorname{conv}}(\operatorname{dent}(A))$ [14, Proposition III.3].

Proposition 2.8. Let $X$ be a Banach space and let $A$ be a closed convex bounded subset of $X$. If $A$ is separable and dentable, then $A$ is $S C D$.

Proof. Since $A$ is separable, so is the set of its denting points, so we may find a countable collection of denting points $\left\{a_{n}: n \in \mathbb{N}\right\}$ of $A$ which is dense in $\operatorname{dent}(A)$. Now, for every $n, m \in \mathbb{N}$, we consider a slice $S_{n, m}$ of $A$ containing $a_{n}$ and having diameter less than $1 / m$. Then, the sequence $\left\{S_{n, m}: n, m \in \mathbb{N}\right\}$ is determining for $A$. Indeed, if $B \subseteq A$ intersects all the $S_{n, m}$, then $a_{n} \in \bar{B}$ for every $n \in \mathbb{N}$, so

$$
A \subseteq \overline{\operatorname{conv}}(\operatorname{dent}(A))=\overline{\operatorname{conv}}\left(\left\{a_{n}: n \in \mathbb{N}\right\}\right) \subseteq \overline{\operatorname{conv}}(\bar{B})=\overline{\operatorname{conv}}(B) .
$$

We recall that there is the concept of a Radon-Nikodým set (defined in terms of vector measures) which is equivalent to dentability of all its closed convex bounded subsets (see [3, §5] or [6, §2]). 
Example 2.9. Let $X$ be a Banach space and let $A$ be a closed convex bounded separable Radon-Nikodým subset of $X$. Then, $A$ is an SCD set.

The norm $\|\cdot\|$ on a Banach space $X$ is said to be $L U R$ at $x_{0} \in S_{X}$ if $\lim \left\|x_{n}-x_{0}\right\|=0$ whenever $\left(x_{n}\right)_{n \in \mathbb{N}} \subseteq B_{X}$ is such that $\lim \left\|x_{n}+x_{0}\right\|=2$. If the norm is LUR at each point of $S_{X}$, we say that $X$ (or its norm) is $L U R$ (see [11, Chapter II] for background). It is clear that every point in the unit sphere of a Banach space $X$ with a LUR norm is denting, so, in this case, $B_{X}$ is dentable.

Example 2.10. Let $X$ be a separable Banach space with an LUR norm. Then, $B_{X}$ is SCD.

It is well known that every separable Banach space admits an LUR renorming (see [11, Theorem II.2.6.]). Therefore, the following result follows immediately from Proposition 2.8 .

Example 2.11. Every separable Banach space $X$ admits an equivalent norm $|\cdot|$ such that $B_{(X,|\cdot|)}$ is an SCD set.

Our second family of elementary examples of SCD sets deals with the so-called Asplund property, a concept related to differentiability of convex continuous functions, which can be equivalently reformulated in terms of separability and duality [6. §5]. A separable closed convex bounded subset $A$ of a Banach space $X$ has the Asplund property if and only if the semi-normed space $\left(X^{*}, \rho_{A}\right)$ is separable, where

$$
\rho_{A}\left(x^{*}\right)=\sup \left\{\left|x^{*}(a)\right|: a \in A\right\} \quad\left(x^{*} \in X^{*}\right) .
$$

Of course, separable closed convex bounded subsets of Asplund spaces have the Asplund property.

Example 2.12. Let $X$ be a Banach space and let $A$ be a closed convex bounded subset of $X$. If $A$ is separable and has the Asplund property, then $A$ is SCD.

Proof. We take a $\rho_{A}$-dense countable family $\left\{x_{n}^{*}: n \in \mathbb{N}\right\}$ in $\left(X^{*}, \rho_{A}\right)$, and consider the slices

$$
S_{n, m}=S\left(A, x_{n}^{*}, 1 / m\right) \quad(n, m \in \mathbb{N}) .
$$

We are done by just proving that if $\left\{v_{n, m}: n, m \in \mathbb{N}\right\}$ satisfies the fact that $v_{n, m} \in S_{n, m}$ for every $n, m \in \mathbb{N}$, then

$$
A \subseteq \overline{\operatorname{conv}}\left(\left\{v_{n, m}: n, m \in \mathbb{N}\right\}\right) .
$$

Indeed, suppose to the contrary that there are $a \in A, x^{*} \in X^{*}$, and $\delta>0$ such that

$$
\operatorname{Re} x^{*}(a)>\sup _{n, m} \operatorname{Re} x^{*}\left(v_{n, m}\right)+\delta .
$$

Now, we may find $N \in \mathbb{N}$ such that $\rho_{A}\left(x_{N}^{*}-x^{*}\right)<\delta / 2$, and so

$$
\begin{aligned}
& \operatorname{Re} x_{N}^{*}(a)+\delta / 2>\operatorname{Re} x^{*}(a)>\sup _{n, m} \operatorname{Re} x^{*}\left(v_{n, m}\right)+\delta \\
& \quad \geqslant \sup _{m} \operatorname{Re} x^{*}\left(v_{N, m}\right)+\delta>\sup _{m} \operatorname{Re} x_{N}^{*}\left(v_{N, m}\right)+\delta / 2=\sup \operatorname{Re} x_{N}^{*}(A)+\delta / 2,
\end{aligned}
$$

a contradiction.

We now show that there are convex bounded subsets of separable Banach spaces which are not SCD. 
Example 2.13. Let $X$ be a separable Banach space with the Daugavet property. Then, $B_{X}$ is not an SCD set. In particular, $B_{C[0,1]}$ and $B_{L_{1}[0,1]}$ are not SCD sets.

Proof. Fix $x_{0} \in S_{X}$ and an arbitrary sequence of slices $\left(S_{n}\right)_{n \in \mathbb{N}}$. We will get the result by showing that there is a sequence $\left(x_{n}\right)_{n \in \mathbb{N}}$ such that $x_{n} \in S_{n}$ for every $n \in \mathbb{N}$ and such that $x_{0} \notin \overline{\operatorname{lin}\left\{x_{n}: n \in \mathbb{N}\right\}}$. To do so, we use [19, Lemma 2.8], which says, in particular, that for every finite-dimensional subspace $Y \subseteq X$, every $\varepsilon>0$, and every slice $S$ of $B_{X}$, there is an $x \in S$ such that

$$
\|y+t x\| \geqslant(1-\varepsilon)(\|y\|+|t|) \quad \forall y \in Y .
$$

Using this result, one can select inductively elements $x_{n} \in S_{n}, n \in \mathbb{N}$, in such a way that

$$
\left\|y+t x_{n}\right\| \geqslant\left(1-\frac{1}{4^{n}}\right)(\|y\|+|t|) \quad\left(y \in \operatorname{lin}\left\{x_{k}: k<n\right\}\right) .
$$

Then, $\left\{x_{n}: n=0,1, \ldots\right\}$ form a sequence equivalent to the unit vector basis of $\ell_{1}$, so $x_{0}$ is not in the closure of $\operatorname{lin}\left\{x_{n}: n \in \mathbb{N}\right\}$, as desired.

For the case of $C[0,1]$, it is possible to give a direct proof without using the Daugavet property, which we include here for the sake of completeness.

Example 2.14. If $K$ is an uncountable metrizable compact space, then the unit ball of $C(K)$ is not an SCD set.

Proof. Let $\mathcal{M}$ be a maximal family of mutually orthogonal continuous measures in $C(K)^{*}$. This induces a decomposition of $C(K)^{*}$ as

$$
C(K)^{*}=\left[\bigoplus_{\mu \in \mathcal{M}} L^{1}(\mu)\right]_{\ell_{1}} \oplus_{1} \ell_{1}(K),
$$

where $\ell_{1}(K)$ is the family of all discrete measures (see [1, pp. 84-85], for instance). As a consequence, we have

$$
C(K)^{* *}=\left[\bigoplus_{\mu \in \mathcal{M}} L^{\infty}(\mu)\right]_{\ell_{\infty}} \oplus_{\infty} \ell_{\infty}(K) .
$$

Let us write the slices of $B_{C[0,1]}$ in the form

$$
U[\nu, \alpha]=\left\{x \in B_{C(K)}: \operatorname{Re} \nu(x)>\alpha\right\},
$$

where $\nu \in C(K)^{*},\|\nu\|=1$, and $-1<\alpha<1$. Suppose, for the sake of contradiction, that there exists a countable family of slices $\mathcal{B}_{0}$ such that every other slice contains one from the family. Then, for every $\mu \in \mathcal{M}$, there exists $V_{\mu}=U\left[\nu_{\mu}, \alpha_{\mu}\right] \in \mathcal{B}_{0}$ such that $V_{\mu} \subseteq U[\mu, 0]$.

Now, for each $\nu \in C(K)^{*}$ we write

$$
\operatorname{Supp}_{\mathcal{M}}(\nu)=\{\mu \in \mathcal{M}: \mu \not \perp \nu\} \text {. }
$$

Notice that this is a countable set which corresponds to the support of $\nu$ in the lefthand side of the decomposition (11). We claim that $\mu \in \operatorname{Supp}_{\mathcal{M}}\left(\nu_{\mu}\right)$ for every $\mu \in \mathcal{M}$. This leads to a contradiction with the facts that $\mathcal{B}_{0}$ and all the sets $\operatorname{Supp}_{\mathcal{M}}\left(\nu_{\mu}\right)$ are countable, while $\mathcal{M}$ is uncountable. Let us prove the claim. Suppose that $\mu \notin \operatorname{Supp}_{\mathcal{M}}\left(\nu_{\mu}\right)$, and let $g$ be an element of the unit ball of $C(K)^{* *}$ where $\nu_{\mu}$ attains its norm. Consider $f \in L^{\infty}(\mu)$ as the $\mu$-coordinate of $g$ when we view 
$g$ as an element of the $\ell_{\infty}$-sum according to (2). Now let $g^{\prime}$ be the element of $C(K)^{* *}$ obtained from $g$ by changing the $\mu$-coordinate from $f$ to $-f$. This is a new element of the unit ball of $C(K)^{* *}$ which satisfies that $g^{\prime}(\mu)=-g(\mu)$ while $g^{\prime}\left(\nu_{\mu}\right)=g\left(\nu_{\mu}\right)=1$. Hence, for either $h=g$ or $h=g^{\prime}$, we have an element $h$ in the unit ball of $C(K)^{* *}$ such that $h\left(\nu_{\mu}\right)=1$ and $h(\mu)<0$. Since the unit ball of $C(K)$ is dense in the unit ball of $C(K)^{* *}$, it follows that $V_{\mu} \backslash U[\mu, 0] \neq \emptyset$.

Remark 2.15. A subset of an SCD set is not necessarily SCD. Indeed, let $X=$ $C[0,1]$. By Example 2.11, there is an equivalent norm $|\cdot|$ on $X$ such that $A=B_{(X,|\cdot|)}$ is SCD. Now, it is possible to find $\lambda>0$ such that $C=\lambda B_{\left(X,\|\cdot\|_{\infty}\right)}$ is contained in $A$. Finally, $C$ is not SCD by Example 2.13 .

Our next goal is to extend the above preliminary examples to more intriguing ones. We will use the so-called Bourgain's lemma [4, Lemma 5.3] (it was rediscovered in [29]) several times, so we state it for the sake of completeness. We refer the reader to [12, Lemma 7.3] for a reference easier to obtain. We recall that a convex combination of slices of a convex bounded subset $A$ of a Banach space $X$ is a subset of $A$ of the form $\sum_{k=1}^{m} \lambda_{i} S_{i}$, where $\lambda_{i}>0, \sum \lambda_{i}=1$ and the $S_{i}$ 's are slices of $A$.

Lemma 2.16 (Bourgain's lemma). Let $X$ be a Hausdorff locally convex space and let $K \subseteq X$ be closed bounded and convex. Then, every nonempty relatively weakly open subset of $K$ contains a convex combination of slices.

Remark 2.17. The condition of closedness of the set in Bourgain's lemma can be omitted. Indeed, let $A$ be a convex bounded set and let $U$ be a relatively weakly open subset of $A$. We denote by $V$ a relatively weakly open subset of $\bar{A}$ such that $V \cap A=U$. By Bourgain's lemma, there are slices $S_{1}, S_{2}, \ldots, S_{n}$ of $\bar{A}$ and coefficients $\lambda_{k}>0$ of a convex combination, such that $\sum_{1}^{n} \lambda_{k} S_{k} \subseteq V$. Then, $S_{k} \cap A$ are slices of $A$ and $\sum_{1}^{n} \lambda_{k} S_{k} \cap A \subseteq V \cap A=U$.

The first consequence is an easy observation.

Proposition 2.18. In the definition of $S C D$ sets, instead of slices one can take convex combinations of slices. Hence, by Bourgain's lemma above, one can also take relatively weakly open subsets.

Proof. Let $\left\{V_{n}: n \in \mathbb{N}\right\}$ be a determining sequence formed by convex combinations of slices of $A$. Now, for every $n \in \mathbb{N}$, there exists a collection of slices $\left\{S_{n, m}: m=1, \ldots, k_{n}\right\}$ and positive numbers $\left\{\lambda_{n, m}: m=1, \ldots, k_{n}\right\}$ with $\sum_{m=1}^{k_{n}} \lambda_{n, m}=1$, such that $\sum_{m=1}^{k_{n}} \lambda_{n, m} S_{n, m} \subseteq V_{n}$. Then, the collection of slices $\left\{S_{n, m}: n \in \mathbb{N}, 1 \leqslant m \leqslant k_{n}\right\}$ is determining for $A$. Indeed, let $B$ be a subset of $A$ such that $B \cap S_{n, m} \neq \emptyset$ for all $n, m$, and consider $b_{n, m} \in B \cap S_{n, m}$ for every $n, m$. If we take $a_{n}=\sum_{m=1}^{k_{n}} \lambda_{n, m} b_{n, m}$, it is clear that $a_{n} \in \operatorname{conv}(B) \cap V_{n}$. So we know that $\operatorname{conv}(B) \cap V_{n} \neq \emptyset$ for all $n$, which by the assumption gives us that $\overline{\operatorname{conv}}(B) \supseteq A$.

Finally, if $A$ has a determining sequence of relatively weakly open subsets $\left\{V_{n}: n \in \mathbb{N}\right\}$, Bourgain's lemma allows us to find convex combinations of slices inside the $V_{n}$ 's, and the proof above shows that $A$ is SCD. 
The first consequence of this result is that Proposition 2.8 can be extended from dentable sets to huskable sets (the same definition with relatively weakly open sets instead of slices). With very little work, we are going to extend the result to the following more general setting. A closed convex bounded subset $A$ of a Banach space $X$ has small combinations of slices [14, 26] if every slice of $A$ contains convex combinations of slices of $A$ with arbitrarily small diameter.

Theorem 2.19. Let $X$ be a Banach space and let $A$ be a separable closed convex bounded subset of $X$ having small combinations of slices. Then, $A$ is an SCD set.

Proof. By [14, Corollary III.7], for every $x \in A$ and every $\varepsilon>0$, there is a convex combination of slices of $A$ contained in $B(x, \varepsilon)$. Now, we take a countable dense subset $\left\{x_{n}: n \in \mathbb{N}\right\}$ of $A$, and for $(n, m) \in \mathbb{N} \times \mathbb{N}$ we take $V_{n, m}$ a convex combination of slices of $A$ contained in $B\left(x_{n}, 1 / m\right)$. Then, if $B \subseteq A$ intersects all the $V_{n, m}$, it also intersects all the balls $B\left(x_{n}, 1 / m\right)$. Therefore, the set $\left\{x_{n}: n \in \mathbb{N}\right\}$ is contained in $\bar{B}$, and so $A=\overline{\operatorname{conv}}(B)$. Finally, Proposition 2.18 gives us that $A$ is SCD.

RNP sets have small combinations of slices, so the above result extends Example 2.9. Even more, strongly regular sets (in particular, huskable sets and CPCP sets) have small combinations of slices [14, Proposition III.5]. We recall that a closed convex bounded subset $A$ of a Banach space is said to be strongly regular if every nonempty convex subset $L$ of $A$ contains a convex combination of slices of $L$ of arbitrarily small diameter. $A$ has the convex point of continuity property ( $C P C P$ for short) if every convex closed subset $B$ of $A$ contains a weak-to-norm point of continuity of the identity mapping. In this case, for every convex subset $B$ of $A$ and for every $\varepsilon>0$, there is a relatively weakly open subset $C \subseteq B$ with $\operatorname{diam}(C)<\varepsilon$ [5].

Corollary 2.20. Let $X$ be a Banach space and let $A$ be a closed convex bounded subset of $X$. If $A$ is separable and strongly regular, then $A$ is SCD. In particular, separable $C P C P$ sets are $S C D$.

Our next aim is to extend Example 2.12 to sets which do not contain $\ell_{1}$ sequences. We need the following topological definition. By a $\pi$-base of a topological space $(T, \tau)$ we understand a family $\left\{O_{i}: i \in I\right\}$ of nonempty open sets such that every nonempty open subset $O$ of $T$ contains one of the elements of the family. The following result is another consequence of Bourgain's lemma.

Proposition 2.21. Let $X$ be a Banach space and let $A$ be a convex bounded subset of $X$. If $\left(A, \sigma\left(X, X^{*}\right)\right)$ has a countable $\pi$-base, then $A$ is an $S C D$ set.

Proof. Let $\left\{V_{n}: n \in \mathbb{N}\right\}$ be a countable $\pi$-base of $\left(A, \sigma\left(X, X^{*}\right)\right)$. Since slices of $A$ have nonempty weak interior, any of them contains some of the $V_{n}$. But then Proposition 2.2 shows that the sequence $\left\{V_{n}\right\}$ is determining for $A$ and Proposition 2.18 gives that $A$ is SCD.

The main consequence of the above proposition is the following. We recall that an $\ell_{1}$-sequence of a Banach space is just a bounded sequence which is equivalent to the natural basis of $\ell_{1}$

Theorem 2.22. Let $X$ be a Banach space and let $A$ be a separable convex bounded subset of $X$ which contains no $\ell_{1}$-sequences. Then $\left(A, \sigma\left(X, X^{*}\right)\right)$ has a countable $\pi$-base. In particular, $A$ is an $S C D$ set. 
Proof. By [12, Theorem 3.11], $\left(A, \sigma\left(X, X^{*}\right)\right)$ is a relatively compact subset of the space of first Baire class functions on $\left(B_{X^{*}}, \sigma\left(X^{*}, X\right)\right)$, and we can apply [30, Lemma 4] by Todorčević to deduce that $\left(A, \sigma\left(X, X^{*}\right)\right)$ has a $\sigma$-disjoint $\pi$-base (i.e. a $\pi$-base $\left\{V_{i}: i \in I\right\}$ such that $I=\bigcup_{n \in \mathbb{N}} I_{n}$ and each subfamily $\left\{V_{i}: i \in I_{n}\right\}$ is a pairwise disjoint family). Now, it is clear that a $\sigma$-disjoint family of open subsets in a separable space has to be countable. Finally, $A$ is SCD by Proposition 2.21.

This result obviously extends Example 2.12 since Asplund sets cannot contain $\ell_{1}$-sequences.

\section{Slicely COUntably Determined SPaCeS}

Definition 3.1. A separable Banach space $X$ is said to be slicely countably determined (an SCD space for short) if every convex bounded subset of $X$ is an SCD set.

By just using the results of the previous section on SCD sets, we get the main examples of SCD spaces.

\section{Examples 3.2.}

(a) If $X$ is a separable strongly regular space, then $X$ is SCD. In particular, RNP spaces (more generally, CPCP spaces) are SCD.

(b) Separable spaces which do not contain copies of $\ell_{1}$ are SCD. In particular, if $X^{*}$ is separable, then $X$ is SCD.

(c) Both families include reflexive separable spaces, which are then SCD spaces.

With respect to spaces which are not SCD, we only know of the Daugavet spaces.

\section{Examples 3.3.}

(a) If $X$ is a separable Banach space which admits an equivalent renorming with the Daugavet property, then $X$ is not SCD.

(b) In particular, there is a Banach space with the Schur property which is not an SCD space. Indeed, in 21] the existence of a separable space having the Schur property and the Daugavet property at the same time was proved.

Let us state the following immediate observations.

Remarks 3.4.

(a) Every subspace of an SCD space is SCD.

(b) For quotients the situation is different. For instance, $C[0,1]$ is a non-SCD quotient of the SCD space $\ell_{1}$.

Our next aim is to show some stability results for the SCD spaces. The first one is a "three space property". We need the following technical lemma which shows that in Definition 3.1 it suffices to consider sets with nonempty interior.

Lemma 3.5. Let $X$ be a separable Banach space. If every open convex bounded subset of $X$ is $S C D$, then $X$ is $S C D$.

Proof. Our first observation is that our hypothesis forces the fact that every bounded convex subset $A$ of $X$ with nonempty interior is SCD. Indeed, notice that since $A$ is convex, the closure of the interior of $A$ coincides with the closure of $A$, and we may apply Remark 2.7 two times to obtain that $A$ is SCD. 
Now, let $A \subseteq X$ be bounded and convex. Since $X$ is separable, we may find a sequence $\left\{x_{n}: n \in \mathbb{N}\right\} \subseteq A$ which is dense in $A$. Let $\left\{\varepsilon_{n}\right\}_{n \in \mathbb{N}}$ be a sequence of positive reals which tends to zero. For every $n, m \in \mathbb{N}$ fixed, we denote $A_{n, m}=$ $\operatorname{conv}\left(B_{\varepsilon_{m}}\left(x_{n}\right) \cup A\right)$ which clearly contains $A$. Since the interior of $A_{n, m}$ is not empty, we may find a determining sequence $\left\{S_{n, m}^{k}: k \in \mathbb{N}\right\}$ of slices of $A_{n, m}$. Now, from the structure of $A_{n, m}$, it follows that either $S_{n, m}^{k} \cap B_{\varepsilon_{m}}\left(x_{n}\right) \neq \emptyset$ or $S_{n, m}^{k} \cap A \neq \emptyset$. Let $K_{n, m}$ be the set of all indices $k \in \mathbb{N}$ for which $S_{n, m}^{k}$ intersects $A$, and denote $\widetilde{S}_{n, m}^{k}=S_{n, m}^{k} \cap A$ for all $k \in K_{n, m}$, which are clearly slices of $A$. Also note that for every integer $k \notin K_{n, m}$, the slice $S_{n, m}^{k}$ intersects $B_{\varepsilon_{m}}\left(x_{n}\right)$. Finally, the family

$$
\left\{\widetilde{S}_{n, m}^{k}: n, m \in \mathbb{N}, k \in K_{n, m}\right\}
$$

is determining for $A$. Indeed, let $B$ be a subset of $A$ intersecting all the $\widetilde{S}_{n, m}^{k}$ and fix some $\varepsilon>0$. Since the sequence $\left\{x_{n}: n \in \mathbb{N}\right\}$ is dense in $A$, there is an integer $n_{0} \in \mathbb{N}$ and $b \in B$ such that $\left\|b-x_{n_{0}}\right\| \leqslant \frac{\varepsilon}{2}$. Also, there is $m_{0} \in \mathbb{N}$ such that $\varepsilon_{m_{0}} \leqslant \frac{\varepsilon}{2}$, as $\varepsilon_{m} \rightarrow 0$ when $m \rightarrow \infty$. We know that $B$ intersects all $S_{n_{0}, m_{0}}^{k}$ with $k \in K_{n, m}$. On the other hand, we also know that the slice $S_{n_{0}, m_{0}}^{k}$ intersects the ball $B_{\varepsilon_{m_{0}}}\left(x_{n_{0}}\right)$ for every $k \notin K_{n, m}$. Hence we can deduce that the set $B_{n_{0}, m_{0}}=B \cup B_{\varepsilon_{m_{0}}}\left(x_{n_{0}}\right) \subseteq A_{n, m}$ intersects all the $S_{n_{0}, m_{0}}^{k}$ which implies that

$$
\overline{\operatorname{conv}}\left(B_{n_{0}, m_{0}}\right) \supseteq A_{n_{0}, m_{0}} \supseteq A \text {. }
$$

Finally, notice that $B_{\varepsilon_{m_{0}}}\left(x_{n_{0}}\right) \subseteq B_{\frac{\varepsilon}{2}}\left(x_{n_{0}}\right) \subseteq B_{\varepsilon}(b)$, which implies that $B_{n_{0}, m_{0}} \subseteq$ $B+\varepsilon B_{X}$. Therefore, we can state that $\overline{\operatorname{conv}}\left(B+\varepsilon B_{X}\right) \supseteq A$, and the arbitrariness of $\varepsilon$ gives us the fact that $\overline{\operatorname{conv}}(B) \supseteq A$.

We may now state the promised stability result.

Theorem 3.6. Let $X$ be a Banach space with a subspace $Z$ such that $Z$ and $Y=$ $X / Z$ are $S C D$ spaces. Then, $X$ is also an $S C D$ space.

Proof. We denote by $q: X \longrightarrow Y=X / Z$ the quotient map. Let us show that every open convex bounded subset $A \subseteq X$ is SCD, and then Lemma 3.5 will imply that $X$ is SCD. To do so, as $X$ is separable since $Y$ and $Z$ are, and separability is a three-space property (see $[9$, Theorem 2.4 .h]), we only need to find, for every point $a \in A$, a sequence of weakly open subsets such that whenever $B \subseteq A$ intersects every member of the sequence, then $a \in \overline{\operatorname{conv}}(B)$ (see Remark 2.4). We fix some $a \in A$ and denote $A_{a}=\{x \in A: q(x)=q(a)\}$. Then, $A_{a}$ is affine isomorphic to an open convex bounded subset of $Z$ which is an SCD space (indeed, $A_{a}=(Z+a) \cap A$ ). It follows that there is a determining sequence $\left\{S_{n}\right\}$ of slices of $A_{a}$. Let $\left\{\widetilde{S}_{n}\right\}$ be their extensions to $A$. For every $n \in \mathbb{N}$, consider $q\left(\widetilde{S}_{n}\right) \subseteq Y$, which is open bounded and convex (its openness is a consequence of the Open Mapping Theorem). Now, as long as $Y$ is $\mathrm{SCD}$, we may find a determining sequence $\left\{S_{n, m}: m \in \mathbb{N}\right\}$ of slices of $q\left(\widetilde{S}_{n}\right)$. Let $V_{n, m}=\widetilde{S}_{n} \cap q^{-1}\left(S_{n, m}\right)$ for every $n, m \in \mathbb{N}$. It is easy to see that the sets $V_{n, m}$ are relatively weakly open. We will now prove that they are the sets we need.

Let $B \subseteq A$ be convex and such that $B \cap V_{n, m} \neq \emptyset$ for all $n, m \in \mathbb{N}$. Fix some $\varepsilon>0$ and denote $B_{\varepsilon}=\{x \in A: \operatorname{dist}(x, B)<\varepsilon\}$. Evidently, $B_{\varepsilon}$ is an open convex set intersecting all the $V_{n, m}$. Having fixed $n \in \mathbb{N}$, we have that

$$
B_{\varepsilon} \cap V_{n, m}=B_{\varepsilon} \cap \widetilde{S}_{n} \cap q^{-1}\left(S_{n, m}\right) \neq \emptyset,
$$


SO

$$
q\left(B_{\varepsilon} \cap \widetilde{S}_{n}\right) \cap S_{n, m} \neq \emptyset
$$

and the choice of $S_{n, m}$ allows us to obtain that

$$
\overline{\operatorname{conv}}\left(q\left(B_{\varepsilon} \cap \widetilde{S}_{n}\right)\right)=\overline{q\left(B_{\varepsilon} \cap \widetilde{S}_{n}\right)} \supseteq q\left(\widetilde{S}_{n}\right) .
$$

Notice that $B_{\varepsilon} \cap \widetilde{S}_{n}$ is open and convex, hence, so is $q\left(B_{\varepsilon} \cap \widetilde{S}_{n}\right)$. This implies that the interior of the set $\overline{q\left(B_{\varepsilon} \cap \widetilde{S}_{n}\right)}$ coincides with $q\left(B_{\varepsilon} \cap \widetilde{S}_{n}\right)$. Now, using the fact that $q\left(\widetilde{S}_{n}\right)$ is open, we obtain that

$$
q\left(B_{\varepsilon} \cap \widetilde{S}_{n}\right) \supseteq q\left(\widetilde{S}_{n}\right)
$$

and, in particular, $q\left(B_{\varepsilon} \cap \widetilde{S}_{n}\right) \ni q(a)$. This means that there exists $x_{n} \in B_{\varepsilon} \cap \widetilde{S}_{n}$, such that $q\left(x_{n}\right)=q(a)$, i.e. that $x_{n} \in B_{\varepsilon} \cap S_{n}$. Since $B_{\varepsilon} \subseteq A$ and $\left\{S_{n}\right\}$ is a determining sequence for $A_{a}$, we get that $B_{\varepsilon} \supseteq A_{a}$. Finally, the arbitrariness of $\varepsilon$ implies that $\bar{B} \supseteq A_{a} \ni a$.

Let us state two immediate consequences of this result.

Corollary 3.7. Let $X$ be a separable Banach space which is not SCD.

(a) $X$ contains copies of $\ell_{1}$, and the quotient of $X$ over any copy of $\ell_{1}$ also contains $\ell_{1}$.

(b) Consequently, for every $\ell_{1}$ subspace $Y_{1}$ of $X$, there is another $\ell_{1}$ subspace $Y_{2}$ such that $Y_{1}$ and $Y_{2}$ are mutually complemented in the closed linear span of $Y_{1}+Y_{2}$ (i.e. $\overline{Y_{1}+Y_{2}}=Y_{1}+Y_{2}=Y_{1} \oplus Y_{2}$ ). In particular, $Y_{1} \cap Y_{2}=0$.

Proof. (a) is immediate from the above theorem and Theorem 2.22, (b) follows from (a) and the "lifting" property of $\ell_{1}$ [22, Proposition 2.f.7].

One may wonder whether item (b) of the above corollary can actually be a characterization of those separable Banach spaces which are not SCD. This is not the case, as the following remark shows.

Remark 3.8. The space $X=\ell_{2}\left(\ell_{1}\right)$ (which is an SCD space; even more, it has the RNP) has the following property: it contains isomorphic copies of $\ell_{1}$, and for every $\ell_{1}$ subspace $Y \subseteq X$ there is another $\ell_{1}$ subspace $Z \subseteq X$, such that $Z$ and $Y$ are mutually complemented in the closed linear span of $Y+Z$.

Proof. Let $\left\{X_{n}\right\}_{n=1}^{\infty}$ be a sequence of isometric copies of $\ell_{1}$. Then, $X$ is isometric to the $\ell_{2}$ direct sum of the spaces $X_{n},\left[\bigoplus_{n \in \mathbb{N}} X_{n}\right]_{\ell_{2}}$. Fix an $\ell_{1}$-subspace $Y \subseteq X$ and let us prove that some of the $X_{n}$ can be taken as $Z$. Assume to the contrary that for every $n \in \mathbb{N}$

$$
\inf \left\{\|y-x\|: y \in S_{Y}, x \in X_{n}\right\}=0 .
$$

Then, for every $n \in \mathbb{N}$ there are $y_{n} \in S_{Y}$ and $x_{n} \in X_{n}$ with $\left\|y_{n}-x_{n}\right\|<10^{-n}$. Since $\left(x_{n}\right)$ forms a bounded sequence of disjoint elements, $\left(x_{n}\right) \longrightarrow 0$ in the weak topology. But then $\left(y_{n}\right) \longrightarrow 0$ in the weak topology as well, which is impossible since $\left(y_{n}\right) \subseteq S_{Y}$ and $Y$ has the Schur property.

Corollary 3.9. Let $X_{1}, \ldots, X_{n}$ be SCD Banach spaces. Then, $X_{1} \oplus \cdots \oplus X_{n}$ is $S C D$. 
Our next goal is to deal with infinite sums. To do so, we need to recall the concept of unconditional sums. Given a sequence $\left\{\left(X_{n},\|\cdot\|_{n}\right): n \in \mathbb{N}\right\}$ of Banach spaces and a Banach space $E$ of sequences whose norm satisfies

$$
\left\|\left(t_{i}\right)\right\|_{E}=\left\|\left(\left|t_{i}\right|\right)\right\|_{E} \quad\left(\left(t_{i}\right) \in E\right),
$$

we denote by $\left[\bigoplus_{n \in \mathbb{N}} X_{n}\right]_{E}$ the Banach space of all sequences $\left(x_{n}\right) \in \prod_{n=1}^{\infty} X_{n}$, so that

$$
\left\|\left(x_{n}\right)\right\|=\left\|\left(\left\|x_{n}\right\|_{n}\right)\right\|_{E}<\infty .
$$

Theorem 3.10. Let $\left\{X_{n}: n \in \mathbb{N}\right\}$ be a sequence of $S C D$ spaces and let $E$ be a Banach space of sequences whose canonical basis is a 1-unconditional and shrinking basis (i.e. $E$ does not contain copies of $\ell_{1}$ ). Then, $X=\left[\bigoplus_{n \in \mathbb{N}} X_{n}\right]_{E}$ is also an SCD space.

Proof. For every $m \in \mathbb{N}$, we denote

$$
Y_{m}=\left[X_{1} \oplus X_{2} \oplus \cdots \oplus X_{m} \oplus 0 \oplus 0 \oplus \cdots\right]_{E} \subseteq X
$$

and let $P_{m}: X \longrightarrow Y_{m}$ be the natural projection. Let $A$ be a convex bounded subset of $X$. Now, for every $m \in \mathbb{N}, P_{m}(A)$ is a convex bounded subset of $Y_{m}$, which is an SCD space by Corollary [3.9. Hence, there is a determining sequence $\left\{S_{m, k}: k \in \mathbb{N}\right\}$ of slices of $P_{m}(A)$. Consider $\widetilde{S}_{m, k}=P_{m}^{-1}\left(S_{m, k}\right) \cap A$. We will prove that $\left\{\widetilde{S}_{m, k}: k, m \in \mathbb{N}\right\}$ is a determining countable collection of slices of $A$.

Let $B$ be a subset of $A$ intersecting all the $\widetilde{S}_{m, k}$. We fix an arbitrary point $a \in A$, and we will prove that $a \in \overline{\operatorname{conv}}(B)$. Since $B$ intersects all the $\widetilde{S}_{m, k}, P_{m}(B)$ intersects $S_{m, k}$ for every integer $k$. It follows that $\overline{\operatorname{conv}}\left(P_{m}(B)\right) \supseteq P_{m}(A)$. In particular, $\overline{\operatorname{conv}}\left(P_{m}(B)\right) \ni P_{m}(a)$. That means that there exists $b_{m} \in \operatorname{conv} B$ such that $\left\|P_{m}\left(b_{m}-a\right)\right\|<\frac{1}{m}$. Then, it is easy to see that $b_{m}$ tends to $a$ coordinate-wise. But since the canonical basis of $E$ is at the same time a shrinking basis, we obtain that $b_{m}$ tends to $a$ in the weak topology. So we can apply Mazur's theorem and obtain a sequence $\left\{b_{m}^{\prime}\right\}$ with $b_{m}^{\prime} \in \operatorname{conv}\left(\left\{b_{k}: k \geqslant m\right\}\right) \subseteq \operatorname{conv}(B)$ which tends to $a$ in the norm topology. But this exactly means that $a \in \overline{\operatorname{conv}}(B)$, which was to be proved.

The next result deals with unconditional sums when the natural basis of $E$ is boundedly complete. Its proof, which is more bulky than the one above, needs a preliminary result which can be of independent interest.

Let $X$ be a Banach space, $A$ be a convex set in $X$ and $\varepsilon$ be a positive real. A point $a \in A$ is called an $\varepsilon$-accessible point of $A$ if there is a sequence $\left\{V_{n}: n \in \mathbb{N}\right\}$ of relatively weakly-open subsets of $A$, such that for every $B \subseteq A$, if $B$ intersects all the $V_{n}$, then $\operatorname{dist}(a, \operatorname{conv} B)<\varepsilon$.

Lemma 3.11. Let $X$ be a Banach space and let $A$ be a separable convex bounded subset of $X$. Suppose that for every convex $C \subseteq A$ and every $\varepsilon>0$, there is an $\varepsilon$-accessible point in $C$. Then, $A$ is an $S C D$ set.

Proof. Notice that, since $A$ is separable, to prove this lemma it is enough to show that for every $\varepsilon>0$, the set $A_{\varepsilon}$ of $\varepsilon$-accessible points of $A$ is dense in $A$. Since $A_{\varepsilon}$ is convex, it is enough to show that $A_{\varepsilon}$ is weakly dense in $A$. Fix some convex relatively weakly-open subset $V \subseteq A$. By the assumption, there is an $\varepsilon$-accessible point of $V$. But this point is also an $\varepsilon$-accessible point of $A$ since $V$ is relatively weakly-open. 
We are now able to state and prove the second result for unconditional sums.

Theorem 3.12. Let $\left\{X_{n}: n \in \mathbb{N}\right\}$ be a sequence of $S C D$ spaces and let $E$ be a space of sequences whose natural basis is a 1-unconditional and boundedly complete basis (i.e. $E$ does not contain isomorphic copies of $c_{0}$ ). Then, $X=\left[\bigoplus_{n \in \mathbb{N}} X_{n}\right]_{E}$ is an $S C D$ space.

Proof. Let a convex bounded subset $A$ of $X$ and $\varepsilon>0$ be fixed. Consider the subset

$$
A_{E}=\left\{\left(a_{n}\right)_{n \in \mathbb{N}} \in E: \exists x=\left(x_{n}\right)_{n \in \mathbb{N}} \in A \text { with }\left\|x_{n}\right\|=\left|a_{n}\right| \text { for all } n \in \mathbb{N}\right\} .
$$

Since $A_{E}$ is a bounded subset of a space with the RNP, there are a functional $b=\left(b_{n}\right)_{n \in \mathbb{N}} \in E^{*}$ and a positive number $\alpha$ such that the slice

$$
S\left(A_{E}\right)=\left\{\left(a_{n}\right)_{n \in \mathbb{N}} \in A_{E}: \sum_{n \in \mathbb{N}} b_{n} a_{n}>\alpha\right\}
$$

has diameter smaller than $\varepsilon / 4$. Taking into account that $A_{E}$ is symmetric, we may assume that $b_{n} \geqslant 0$ (the slice of $A_{E}$ defined by $|b|=\left(\left|b_{n}\right|\right)_{n \in \mathbb{N}}$ is isometric to $\left.S\left(A_{E}\right)\right)$. Fix an $x \in A$ with $\left(\left\|x_{n}\right\|\right)_{n \in \mathbb{N}} \in S\left(A_{E}\right)$ and pick $x_{n}^{*} \in S_{X_{n}^{*}}$ such that $x_{n}^{*}\left(x_{n}\right)=\left\|x_{n}\right\|$. Write $f_{n}=b_{n} x_{n}^{*}, f=\left(f_{n}\right)_{n \in \mathbb{N}} \in X^{*}$. We claim that for the slice

$$
S=\left\{\left(x_{n}\right)_{n \in \mathbb{N}} \in A: \sum_{n \in \mathbb{N}} f_{n}\left(x_{n}\right)>\alpha\right\}
$$

there is an $m \in \mathbb{N}$ with the following property:

$$
\left\|\left(0, \ldots, 0, y_{m+1}, y_{m+2}, \ldots\right)\right\|<\frac{\varepsilon}{2} \quad \text { for all }\left(y_{n}\right)_{n \in \mathbb{N}} \in S .
$$

To show this, it is sufficient to select $m$ in such a way that

$$
\left\|\left(0, \ldots, 0, x_{m+1}, x_{m+2}, \ldots\right)\right\|<\varepsilon / 4
$$

and to use the fact that $\operatorname{diam} S\left(A_{E}\right)<\varepsilon / 4$. In fact, with such a choice of $m$ we get

$$
\begin{aligned}
\left\|\left(0, \ldots, 0, y_{m+1}, y_{m+2}, \ldots\right)\right\| & \\
= & \left\|\left(0, \ldots, 0,\left\|y_{m+1}\right\|,\left\|y_{m+2}\right\|, \ldots\right)\right\| \\
\leqslant & \left\|\left(0, \ldots, 0,\left\|x_{m+1}\right\|,\left\|x_{m+2}\right\|, \ldots\right)\right\| \\
& \quad+\left\|\left(0, \ldots, 0,\left|\left\|x_{m+1}\right\|-\left\|y_{m+1}\right\|\right|,\left|\left\|x_{m+2}\right\|-\left\|y_{m+2}\right\|\right|, \ldots\right)\right\| \\
\leqslant & \frac{\varepsilon}{4}+\left\|\left(\left|\left\|x_{1}\right\|-\left\|y_{1}\right\|\right|,\left|\left\|x_{2}\right\|-\left\|y_{2}\right\|\right|, \ldots\right)\right\| \leqslant \frac{\varepsilon}{2} .
\end{aligned}
$$

Let us prove that $x$ is an $\varepsilon$-accessible point of $A$. Consider

$$
Y_{m}=\left[X_{1} \oplus X_{2} \oplus \cdots \oplus X_{m} \oplus 0 \oplus 0 \oplus \cdots\right]_{E} \subseteq X
$$

and $P_{m}: X \longrightarrow Y_{m}$ as the natural projection. By Corollary 3.9, $Y_{m}$ is an SCD space and, since $P_{m}(S)$ is a convex bounded set in $Y_{m}$, there exists a determining sequence $\left\{S_{n}: n \in \mathbb{N}\right\}$ of slices of $P_{m}(S)$. Notice that $Y_{m}^{*}$ isometrically embeds into $X^{*}$. For every integer $n \in \mathbb{N}$, we consider $\widetilde{S}_{n}=P_{m}^{-1} S_{n} \cap S$, which is a slice of $S$ and, obviously, relatively weakly-open in $A$. Let $B$ be a subset of $A$ which intersects all the $\widetilde{S}_{n}$. We will now prove that then $\operatorname{dist}(x, \operatorname{conv}(B))<\varepsilon$. Since $B$ intersects all the $\widetilde{S}_{n}$, we can find a sequence $\left\{y_{n}\right\} \subseteq B$, such that $y_{n} \in \widetilde{S}_{n}$ for every $n \in \mathbb{N}$. This implies that $P_{m}\left(y_{n}\right) \in S_{n}$ for all $n \in \mathbb{N}$, and so $\overline{\operatorname{conv}}\left(\left\{P_{m}\left(y_{n}\right): n \in \mathbb{N}\right\}\right) \supseteq P_{m}(S)$. 
In particular, $P_{m}(x) \in \overline{\operatorname{conv}}\left(\left\{P_{m}\left(y_{n}\right): n \in \mathbb{N}\right\}\right)$. But (3) gives us that the $m$-th tails of $x$ and of all the $y_{n}$ are small, that is,

$$
\left\|x-P_{m}(x)\right\|<\frac{\varepsilon}{2} \quad \text { and } \quad\left\|y_{n}-P_{m}\left(y_{n}\right)\right\|<\varepsilon / 2 \quad(\text { for all } n \in \mathbb{N}) .
$$

This gives us that $\operatorname{dist}(a, \operatorname{conv}(B))<\varepsilon$, and the proof is complete.

An immediate consequence is the following.

Example 3.13. The spaces $c_{0}\left(\ell_{1}\right)$ and $\ell_{1}\left(c_{0}\right)$ are SCD.

This result, together with those results of section 2, gives us the following examples.

Example 3.14. The spaces $c_{0} \otimes_{\varepsilon} c_{0}, c_{0} \otimes_{\pi} c_{0}, c_{0} \otimes_{\varepsilon} \ell_{1}, c_{0} \otimes_{\pi} \ell_{1}, \ell_{1} \otimes_{\varepsilon} \ell_{1}$, and $\ell_{1} \otimes_{\pi} \ell_{1}$ are SCD. Indeed, it is well known that $c_{0} \otimes_{\varepsilon} c_{0} \equiv c_{0}, c_{0} \otimes_{\varepsilon} \ell_{1} \equiv c_{0}\left(\ell_{1}\right)$, $c_{0} \otimes_{\pi} \ell_{1} \equiv \ell_{1}\left(c_{0}\right)$, and $\ell_{1} \otimes_{\pi} \ell_{1} \equiv \ell_{1}$ (see [27, Examples 2.19 and 3.3], for instance), so these cases are clear from the above example. For the remaining cases, just observe that $\left[c_{0} \otimes_{\pi} c_{0}\right]^{*} \equiv \ell_{1} \otimes_{\varepsilon} \ell_{1}\left(\right.$ since $\left[c_{0} \otimes_{\pi} c_{0}\right]^{*} \equiv L\left(c_{0}, \ell_{1}\right)$ [27, p. 24], $K\left(c_{0}, \ell_{1}\right) \equiv \ell_{1} \otimes_{\varepsilon} \ell_{1}$ 27. Corollary 4.13] and $K\left(c_{0}, \ell_{1}\right)=L\left(c_{0}, \ell_{1}\right)$ since $\ell_{1}$ has the Schur property and $c_{0}^{*}$ is separable), so $c_{0} \otimes_{\pi} c_{0}$ is Asplund and $\ell_{1} \otimes_{\varepsilon} \ell_{1}$ has the RNP.

Since for $X$ and $Y$ being $c_{0}$ or $\ell_{1}$ one has $K(X, Y) \equiv X^{*} \otimes_{\varepsilon} Y$ [27, Corollary 4.13], the following examples follow.

Example 3.15. The spaces $K\left(c_{0}\right)$ and $K\left(c_{0}, \ell_{1}\right)$ are SCD. The spaces $K\left(\ell_{1}\right)$ and $K\left(\ell_{1}, c_{0}\right)$ contain $\ell_{\infty}$, and so they are not separable, all the more not SCD.

Another example in this line is the following.

Example 3.16. The spaces $\ell_{2} \otimes_{\pi} \ell_{2} \equiv \mathcal{L}_{1}\left(\ell_{2}\right)$ and $\ell_{2} \otimes_{\varepsilon} \ell_{2} \equiv K\left(\ell_{2}\right)$ are SCD. Indeed, the first space has the RNP and the second is an Asplund space.

\section{An application to SPACES With NUMerical IndeX 1}

Our aim in this section is to show that SCD spaces with the alternative Daugavet property are lush. To get such a result, we need to establish a characterization of the alternative Daugavet property which can be of independent interest. We first recall a previous characterization in terms of slices.

Lemma 4.1 ([25, Proposition 2.1]). A Banach space $X$ has the alternative Daugavet property if and only if for every $x \in S_{X}$, every $\varepsilon>0$ and every slice $S$ of $B_{X}$, there is a $y \in S$ such that $\max _{\theta \in \mathbb{T}}\|x+\theta y\|>2-\varepsilon$.

We need some notation. Denote $K\left(X^{*}\right)$ as the intersection of $S_{X^{*}}$ with the weak*-closure in $X^{*}$ of $\operatorname{ext}\left(B_{X^{*}}\right)$, and for every slice $S$ of $B_{X}$ and every $\varepsilon>0$, we write

$$
\begin{aligned}
D(S, \varepsilon) & =\left\{y^{*} \in K\left(X^{*}\right): S \cap \mathbb{T} S\left(B_{X}, y^{*}, \varepsilon\right) \neq \emptyset\right\} \\
& =\left\{y^{*} \in K\left(X^{*}\right): S \cap \overline{\operatorname{aconv}}\left(S\left(B_{X}, y^{*}, \varepsilon\right)\right) \neq \emptyset\right\},
\end{aligned}
$$

which is relatively weak*-open in $K\left(X^{*}\right)$. Here is the promised characterization of the alternative Daugavet property.

Proposition 4.2. For a Banach space $X$, the following assertions are equivalent:

(i) $X$ has the alternative Daugavet property. 
(ii) For every $x \in S_{X}$, every $\varepsilon>0$ and every slice $S \subseteq B_{X}$, there is $y^{*} \in K\left(X^{*}\right)$ such that $x \in S\left(B_{X}, y^{*}, \varepsilon\right)$ and $S \cap \mathbb{T} S\left(B_{X}, y^{*}, \varepsilon\right) \neq \emptyset$.

(iii) For every $x \in S_{X}$, every $\varepsilon>0$ and every slice $S \subseteq B_{X}$, there is $y^{*} \in D(S, \varepsilon)$ such that $x \in S\left(B_{X}, y^{*}, \varepsilon\right)$.

(iv) For every $\varepsilon>0$ and every slice $S \subseteq B_{X}$, the set $D(S, \varepsilon)$ is weak ${ }^{*}$-dense in $K\left(X^{*}\right)$.

(v) For every $\varepsilon>0$ and every sequence $\left\{S_{n}: n \in \mathbb{N}\right\}$ of slices of $B_{X}$, the set $\bigcap_{n \in \mathbb{N}} D\left(S_{n}, \varepsilon\right)$ is weak ${ }^{*}$-dense in $K\left(X^{*}\right)$.

Proof. The implications (i) $\Longleftrightarrow$ (ii) $\Longleftrightarrow$ (iii) are easy consequences of Lemma 4.1 .

(iii) $\Longrightarrow$ (iv). To show weak ${ }^{*}$-density of $D(S, \varepsilon)$ in $K\left(X^{*}\right)$, it is sufficient to demonstrate that the weak* closure of $D(S, \varepsilon)$ contains every extreme point $x^{*}$ of $S_{X^{*}}$. Since weak ${ }^{*}$-slices form a base of neighborhoods of $x^{*}$ in $B_{X^{*}}$, it is sufficient to prove that every weak ${ }^{*}$-slice $S\left(B_{X^{*}}, x, \delta\right)$ with $\delta \in(0, \varepsilon)$ and $x \in S_{X}$ intersects $D(S, \varepsilon)$, i.e. that there is a point $y^{*} \in D(S, \varepsilon)$, such that $y^{*} \in S\left(B_{X^{*}}, x, \delta\right)$. But we know that there is a point $y^{*} \in D(S, \delta) \subseteq D(S, \varepsilon)$, such that $x \in S\left(B_{X}, y^{*}, \delta\right)$, which means that $y^{*} \in S\left(B_{X^{*}}, x, \delta\right)$.

(iv) $\Longrightarrow$ (iii). If $D(S, \varepsilon)$ is weak*-dense in $K\left(X^{*}\right)$, then for every $x \in S_{X}$ there is a $y^{*} \in D(S, \varepsilon)$ such that $x \in S\left(B_{X}, y^{*}, \varepsilon\right)$.

The remaining equivalence (iv) $\Longleftrightarrow(\mathrm{v})$ follows from the fact that $D(S, \varepsilon)$ is not only weak ${ }^{*}$-dense but also weak*-open, and $K\left(X^{*}\right)$ is a Baire space (indeed, let $K^{\prime}\left(X^{*}\right)$ be the weak*-closure in $X^{*}$ of $\operatorname{ext}\left(B_{X^{*}}\right)$ which is weak*-compact and observe that

$$
K^{\prime}\left(X^{*}\right) \backslash K\left(X^{*}\right)=\bigcup_{n \in \mathbb{N}}\left[\left(1-\frac{1}{n}\right) B_{X^{*}} \cap K^{\prime}\left(X^{*}\right)\right]
$$

is of the first category in $\left.K^{\prime}\left(X^{*}\right)\right)$. Therefore, Baire's theorem is applicable.

It is possible to give a result analogous to the above one for the Daugavet property. We need to give more notation. For every slice $S$ of $B_{X}$ and every $\varepsilon>0$, we write

$$
\begin{aligned}
\widetilde{D}(S, \varepsilon) & =\left\{y^{*} \in K\left(X^{*}\right): S \cap S\left(B_{X}, y^{*}, \varepsilon\right) \neq \emptyset\right\} \\
& =\left\{y^{*} \in K\left(X^{*}\right): S \cap \overline{\operatorname{conv}}\left(S\left(B_{X}, y^{*}, \varepsilon\right)\right) \neq \emptyset\right\},
\end{aligned}
$$

which is relatively weak ${ }^{*}$-open in $K\left(X^{*}\right)$. The proof of the next result is almost the same as the above one, replacing Lemma 4.1 by [19, Lemma 2.2]. We include it here for future use.

Proposition 4.3. For a Banach space $X$, the following assertions are equivalent:

(i) $X$ has the Daugavet property.

(ii) For every $x \in S_{X}$, every $\varepsilon>0$ and every slice $S \subseteq B_{X}$, there is $y^{*} \in K\left(X^{*}\right)$ such that $x \in S\left(B_{X}, y^{*}, \varepsilon\right)$ and $S \cap S\left(B_{X}, y^{*}, \varepsilon\right) \neq \emptyset$.

(iii) For every $x \in S_{X}$, every $\varepsilon>0$ and every slice $S \subseteq B_{X}$, there is $y^{*} \in \widetilde{D}(S, \varepsilon)$ such that $x \in S\left(B_{X}, y^{*}, \varepsilon\right)$.

(iv) For every $\varepsilon>0$ and every slice $S \subseteq B_{X}$, the set $\widetilde{D}(S, \varepsilon)$ is weak ${ }^{*}$-dense in $K\left(X^{*}\right)$.

(v) For every $\varepsilon>0$ and every sequence $\left\{S_{n}: n \in \mathbb{N}\right\}$ of slices of $B_{X}$, the set $\bigcap_{n \in \mathbb{N}} \widetilde{D}\left(S_{n}, \varepsilon\right)$ is weak ${ }^{*}$-dense in $K\left(X^{*}\right)$.

We are now ready to show the main result of this section. 
Theorem 4.4. Every Banach space $X$ with the alternative Daugavet property whose unit ball is an SCD set is lush. In particular, every SCD space with the alternative Daugavet property is lush.

Proof. Let $\left\{S_{n}: n \in \mathbb{N}\right\}$ be the sequence of slices of $B_{X}$ from the definition of an SCD set. Then, by Proposition $4.2(\mathrm{v})$, for every $\varepsilon>0$ the set $\bigcap_{n \in \mathbb{N}} D\left(S_{n}, \varepsilon\right)$ is weak ${ }^{*}$-dense in $K\left(X^{*}\right)$ (which is norming). So, for every $x \in S_{X}$ there is $y^{*} \in$ $\bigcap_{n \in \mathbb{N}} D\left(S_{n}, \varepsilon\right)$ such that $x \in S\left(B_{X}, y^{*}, \varepsilon\right)$. According to the definition of $D\left(S_{n}, \varepsilon\right)$, this means that $S_{n} \cap \overline{\operatorname{aconv}}\left(S\left(B_{X}, y^{*}, \varepsilon\right)\right) \neq \emptyset$ for all $n \in \mathbb{N}$. Then, we obtain that $\overline{\operatorname{aconv}}\left(S\left(B_{X}, y^{*}, \varepsilon\right)\right)=B_{X}$, which implies lushness of $X$ [7, Theorem 2.1].

Remark 4.5. Let us observe that in the above proof a (formally) weaker version of an SCD set is used. A convex bounded subset $A$ of a Banach space $X$ is said to be almost slicely countably determined (almost-SCD for short) if there is a sequence $\left\{V_{n}: n \in \mathbb{N}\right\}$ of subsets of $A$ such that for every $B \subseteq A$ intersecting all the $V_{n}$, one has $\overline{\operatorname{aconv}}(B) \supseteq A$. The proof of the above theorem actually shows that every Banach space $X$ with the alternative Daugavet property whose unit ball is an almost-SCD is lush.

Theorem 4.4 has already been known for Asplund spaces and for spaces with the RNP [23, Remark 6], regardless of the separability (necessary for the SCD and so for our result). Our next goal is to particularize Theorem 4.4 to more cases where we are able to remove the separability. The proof of the following results is a consequence of the facts that lushness and the alternative Daugavet property are separably determined (see [7, Theorem 4.2] for the first case and the remark below for the second one).

Remark 4.6. It is shown in [20, Theorem 4.5] that the Daugavet property is separably determined. With a little effort, the proof can be adapted to the alternative Daugavet property: A Banach space $X$ has the alternative Daugavet property if and only if for every separable subspace $Y \subseteq X$ there is a separable subspace $Z \subseteq X$ which contains $Y$ and has the alternative Daugavet property.

Corollary 4.7. Let $X$ be a Banach space with the alternative Daugavet property. If $X$ is strongly regular (in particular, $C P C P$ ), then $X$ is lush.

Corollary 4.8. Let $X$ be a Banach space with the alternative Daugavet property. If $X$ does not contain $\ell_{1}$, then $X$ is lush.

This latter result solves in the positive Problem 32 of [18, and it can be used to prove a necessary isomorphic condition for a real Banach space to have the alternative Daugavet property.

Corollary 4.9. Let $X$ be an infinite-dimensional real Banach space with the alternative Daugavet property. Then, $X^{*}$ contains $\ell_{1}$.

Proof. If $X$ contains $\ell_{1}$, then $X^{*}$ contains a quotient isomorphic to $\ell_{\infty}$, so $X^{*}$ contains $\ell_{1}$ as a quotient and the "lifting" property of $\ell_{1}$ [22, Proposition 2.f.7] gives us $X^{*} \supseteq \ell_{1}$. Otherwise, Corollary 4.8 gives us that $X$ is lush. But the dual of an infinite-dimensional real lush space contains $\ell_{1}$ [16, Corollary 4.9].

In particular, since Banach spaces with numerical index 1 have the alternative Daugavet property, we get the following corollary which answers in the positive Problem 18 of [18. 
Corollary 4.10. Let $X$ be an infinite-dimensional real Banach space with $n(X)=$ 1. Then, $X^{*} \supseteq \ell_{1}$.

Let us comment that very recently it has been shown that there are Banach spaces with numerical index 1 which are not lush [17, so the above result is not covered by [16, Corollary 4.9].

\section{SCD OPERATORS}

Definition 5.1. Let $X$ and $Y$ be Banach spaces. A bounded linear operator $T: X \longrightarrow Y$ is said to be an $S C D$-operator if $T\left(B_{X}\right)$ is an SCD set.

By just recalling the examples of SCD sets and SCD spaces given in sections 2 and 3 , we get the main examples of SCD-operators.

Examples 5.2. Let $X$ and $Y$ be Banach spaces and let $T: X \longrightarrow Y$ be a bounded linear operator such that $T(X)$ is separable.

(a) If $T\left(B_{X}\right)$ has small combinations of slices, then $T$ is an SCD-operator.

(b) In particular, if $T\left(B_{X}\right)$ is a Radon-Nikodým set (i.e. if $T$ is a strong RadonNikodým operator), then $T$ is an SCD-operator.

(c) If $T\left(B_{X}\right)$ does not contain $\ell_{1}$-sequences, then $T$ is an SCD-operator.

(d) In particular, if $T$ does not fix copies of $\ell_{1}$, then $T$ is an SCD-operator. Indeed, if $T\left(B_{X}\right)$ contains an $\ell_{1}$-sequence $\left(T e_{n}\right)_{n \in \mathbb{N}}$ with $e_{n} \in B_{X}(n \in \mathbb{N})$, then as in the proof of the "lifting" property of $\ell_{1}$ [22, Proposition 2.f.7], $Y=\varlimsup\left\{e_{n}: n \in \mathbb{N}\right\}$ is a copy of $\ell_{1}$ and $\left.T\right|_{Y}$ is an isomorphic embedding, a contradiction (see [31, Proposition 1]).

The aim of this section is to show that SCD-operators behave in a very good way with respect to the Daugavet and the alternative Daugavet equations. We start with the best result we can get for the alternative Daugavet property.

Theorem 5.3. Let $X$ be a Banach space with the alternative Daugavet property and let $T \in L(X)$ be an $S C D$-operator. Then, $\max _{\theta \in \mathbb{T}}\|\mathrm{Id}+\theta T\|=1+\|T\|$.

Proof. Without loss of generality, we may assume that $\|T\|=1$. We take a determining sequence $\left\{S_{n}: n \in \mathbb{N}\right\}$ of slices of $T\left(B_{X}\right)$, and we notice that the sets $T^{-1}\left(S_{n}\right) \cap B_{X}$ are slices of $B_{X}$. Given $\varepsilon>0$ fixed, we take $a \in S_{X}$ such that $\|T(a)\|>1-\varepsilon$. Now, Proposition 4.2, v gives us that $\bigcap_{n \in \mathbb{N}} D\left(T^{-1}\left(S_{n}\right), \varepsilon\right)$ is weak*dense in $K\left(X^{*}\right)$ (which is norming for $X$ ), so we may find $y^{*} \in \bigcap_{n \in \mathbb{N}} D\left(T^{-1}\left(S_{n}\right), \varepsilon\right.$ ) such that

$$
\operatorname{Re} y^{*}(T(a)) \geqslant\|T(a)\|-\varepsilon>1-2 \varepsilon .
$$

By the definition of $D\left(T^{-1}\left(S_{n}\right), \varepsilon\right)$, we get that

$$
\overline{\operatorname{aconv}}\left(S\left(B_{X}, y^{*}, \varepsilon\right)\right) \cap T^{-1}\left(S_{n}\right) \neq \emptyset \quad(n \in \mathbb{N}) .
$$

Thus, $T\left(\overline{\operatorname{aconv}}\left(S\left(B_{X}, y^{*}, \varepsilon\right)\right)\right) \cap S_{n} \neq \emptyset$ for all $n \in \mathbb{N}$, and using the fact that $\left\{S_{n}: n \in \mathbb{N}\right\}$ is determining, we deduce that

$$
\overline{T\left(\operatorname{aconv}\left(S\left(B_{X}, y^{*}, \varepsilon\right)\right)\right)}=\overline{\operatorname{aconv}}\left(T\left(\overline{\operatorname{aconv}}\left(S\left(B_{X}, y^{*}, \varepsilon\right)\right)\right)\right) \supseteq T\left(B_{X}\right) .
$$

In particular, $T(a) \in \overline{T\left(\operatorname{aconv}\left(S\left(B_{X}, y^{*}, \varepsilon\right)\right)\right)}$, which means that there is

$$
z \in T\left(\operatorname{aconv}\left(S\left(B_{X}, y^{*}, \varepsilon\right)\right)\right) \quad \text { with }\|T(a)-z\|<\varepsilon,
$$


and it follows from (4) that

$$
\operatorname{Re} y^{*}(z)>1-3 \varepsilon \text {. }
$$

Notice that $z$ can be represented in the following way:

$$
z=T\left(\sum_{k=1}^{m} \lambda_{k} \theta_{k} x_{k}\right)=\sum_{k=1}^{m} \lambda_{k} \theta_{k} T\left(x_{k}\right)
$$

where $x_{k} \in S\left(B_{X}, y^{*}, \varepsilon\right), \theta_{k} \in \mathbb{T}, \lambda_{k} \geqslant 0$ for $k=1, \ldots, m$ and $\sum_{k=1}^{m} \lambda_{k}=1$. Then, it follows from (5) that there exists $k_{0} \in\{1, \ldots, m\}$ such that

$$
\operatorname{Re} y^{*}\left(\theta_{k_{0}} T\left(x_{k_{0}}\right)\right)>1-3 \varepsilon .
$$

Now, since $x_{k_{0}} \in S\left(B_{X}, y^{*}, \varepsilon\right)$, we get that

$$
\operatorname{Re} y^{*}\left(x_{k_{0}}+\theta_{k_{0}} T\left(x_{k_{0}}\right)\right)>2-4 \varepsilon \text {. }
$$

It follows that

$$
\left\|\operatorname{Id}+\theta_{k_{0}} T\right\| \geqslant\left\|x_{k_{0}}+\theta_{k_{0}} T\left(x_{k_{0}}\right)\right\| \geqslant \operatorname{Re} y^{*}\left(x_{k_{0}}+\theta_{k_{0}} T\left(x_{k_{0}}\right)\right)>2-4 \varepsilon .
$$

Finally, the arbitrariness of $\varepsilon$ gives the result.

Remark 5.4. Analogous to the situation described in Remark 4.5 in the above proof we have used a formally weaker property than an SCD-operator. Therefore, the result proved is the following. Let $X$ be a Banach space with the alternative Daugavet property and let $T \in L(X)$ such that $T\left(B_{X}\right)$ is an almost-SCD set. Then, $\max _{\theta \in \mathbb{T}}\|\mathrm{Id}+\theta T\|=1+\|T\|$.

We can easily obtain a version of Theorem 5.3 for operators with nonseparable range which is useful for applications.

Corollary 5.5. Let $X$ be a Banach space with the alternative Daugavet property and let $T \in L(X)$ be such that $T\left(B_{Y}\right)$ is an $S C D$ set for every separable subspace $Y$ of $X$. Then, $\max _{\theta \in \mathbb{T}}\|\mathrm{Id}+\theta T\|=1+\|T\|$.

Proof. We first take a separable subspace $Y_{1}$ of $X$ such that $\left\|\left.T\right|_{Y_{1}}\right\|=\|T\|$. Then, Remark 4.6 provides us with a separable subspace $Y_{2}$ with the alternative Daugavet property which contains $\bigcup_{k=0}^{\infty} T^{k}\left(Y_{1}\right)$. We again apply Remark 4.6 to get a separable subspace $Y_{3}$ with the alternative Daugavet property which contains $\bigcup_{k=0}^{\infty} T^{k}\left(Y_{2}\right)$, and so on. Then, the space $Y=\overline{\bigcup_{n \in \mathbb{N}} Y_{n}}$ is separable, $T$-invariant, $\left\|\left.T\right|_{Y}\right\|=\|T\|$, and it has the alternative Daugavet property (just use Lemma 4.1). Since $T\left(B_{Y}\right)$ is SCD, Theorem 5.3 gives us that

$$
\max _{\theta \in \mathbb{T}}\|\operatorname{Id}+\theta T\| \geqslant \max _{\theta \in \mathbb{T}}\left\|\left.\operatorname{Id}\right|_{Y}+\left.\theta T\right|_{Y}\right\|=1+\left\|\left.T\right|_{Y}\right\|=1+\|T\| .
$$

The following particular cases are especially interesting. The first one solves 18 , Problem 33].

Corollary 5.6. Let $X$ be a Banach space with the alternative Daugavet property and let $T \in L(X)$ be an operator which does not fix copies of $\ell_{1}$. Then,

$$
\max _{\theta \in \mathbb{T}}\|\mathrm{Id}+\theta T\|=1+\|T\| .
$$

Corollary 5.7. Let $X$ be a Banach space with the alternative Daugavet property and let $T \in L(X)$ be an operator such that $T\left(B_{X}\right)$ is strongly regular. Then, $\max _{\theta \in \mathbb{T}}\|\mathrm{Id}+\theta T\|=1+\|T\|$. 
It is possible to show an analogous result to Theorem 5.3 for spaces with the Daugavet property and the Daugavet equation. Actually, it is possible to get a better result. We need some notation and preliminary results. A bounded linear operator $T: X \longrightarrow Y$ between two Banach spaces $X$ and $Y$ is said to be a strong Daugavet operator if for every $x, y \in S_{X}$ and every $\varepsilon>0$, there is an element $z \in S_{X}$ such that

$$
\|x+z\| \geqslant 2-\varepsilon \quad \text { and } \quad\|T y-T z\|<\varepsilon
$$

(see [20, §3] for the definition and the following properties). If $T \in L(X)$ is a strong Daugavet operator and $X$ has the Daugavet property, then $T$ satisfies the Daugavet equation. On the other hand, finite-rank operators from a space with the Daugavet property are strong Daugavet operators. Our next goal is to show that, actually, SCD-operators are strong Daugavet operators.

Proposition 5.8. Let $X$ be a Banach space with Daugavet property, let $Y$ be a Banach space, and let $T: X \longrightarrow Y$ be an SCD-operator. Then, $T$ is a strong Daugavet operator.

Proof. Since $T$ is an SCD-operator, we may find a determining sequence $\left\{S_{n}: n \in\right.$ $\mathbb{N}\}$ of slices of $T\left(B_{X}\right)$, and we notice that the sets $T^{-1}\left(S_{n}\right) \cap B_{X}$ are slices of $B_{X}$. We fix $\varepsilon>0$ and $x, y \in S_{X}$.

Since $X$ has the Daugavet property, Proposition 4.3(v) gives us the fact that $\bigcap_{n \in \mathbb{N}} \widetilde{D}\left(T^{-1}\left(S_{n}\right), \frac{\varepsilon}{2}\right)$ is weak*-dense in $K\left(X^{*}\right)$ (which is norming for $X$ ), so we may find $y^{*} \in \bigcap_{n \in \mathbb{N}} \widetilde{D}\left(T^{-1}\left(S_{n}\right), \frac{\varepsilon}{2}\right)$ such that

$$
x \in S\left(B_{X}, y^{*}, \frac{\varepsilon}{2}\right) .
$$

Then, by the definition of $\widetilde{D}\left(T^{-1}\left(S_{n}\right), \frac{\varepsilon}{2}\right)$, we have that $\overline{S\left(B_{X}, y^{*}, \frac{\varepsilon}{2}\right)} \cap T^{-1}\left(S_{n}\right) \neq \emptyset$ for every $n \in \mathbb{N}$. Thus,

$$
T\left(\overline{S\left(B_{X}, y^{*}, \frac{\varepsilon}{2}\right)}\right) \cap S_{n} \neq \emptyset \quad(n \in \mathbb{N}) .
$$

Now, since the sequence $\left\{S_{n}: n \in \mathbb{N}\right\}$ is determining, we deduce that

$$
T\left(B_{X}\right) \subseteq \overline{\operatorname{conv}} T\left(\overline{S\left(B_{X}, y^{*}, \frac{\varepsilon}{2}\right)}\right)=\overline{T\left(S\left(B_{X}, y^{*}, \frac{\varepsilon}{2}\right)\right)} .
$$

In particular, $T y \in \overline{T\left(S\left(B_{X}, y^{*}, \frac{\varepsilon}{2}\right)\right)}$, which means that there is a $z \in S\left(B_{X}, y^{*}, \frac{\varepsilon}{2}\right)$ such that

$$
\|T y-T z\|<\varepsilon
$$

Since $x \in S\left(B_{X}, y^{*}, \frac{\varepsilon}{2}\right)$ by (6) , we also have that

$$
\|x+z\|>2-\varepsilon
$$

Hence, this $z$ meets all the requirements.

In particular, we obtain the following analogue to Theorem 5.3 .

Corollary 5.9. Let $X$ be a Banach space with the Daugavet property. If $T \in L(X)$ is an SCD-operator, then $\|\mathrm{Id}+T\|=1+\|T\|$.

Our final goal in this section is to get a better result than Proposition 5.8 for a class of operators more restrictive than the SCD-operators. We need some notation. A bounded linear operator $T: X \longrightarrow Y$ between two Banach spaces $X$ and $Y$ is said to be a narrow operator if for every $x^{*} \in X^{*}$, the operator

$$
T \tilde{+} \operatorname{Re} x^{*}: X \longrightarrow Y \oplus_{1} \mathbb{R}, \quad x \longmapsto\left(T x, \operatorname{Re} x^{*}(x)\right)
$$


is a strong Daugavet operator (see [20, $\S 3$ and $\S 4]$ for this definition and the following properties). Equivalently, $T$ is narrow if and only if for every $x, y \in S_{X}$, every $\varepsilon>0$, and every slice $S$ of $B_{X}$ containing $y$, there is an element $z \in S$ such that

$$
\|x+z\| \geqslant 2-\varepsilon \quad \text { and } \quad\|T y-T z\|<\varepsilon .
$$

A narrow operator is strong Daugavet, but the converse result is not true. It is known that strong Radon-Nikodým operators and operators which do not fix copies of $\ell_{1}$ from a Banach space with the Daugavet property are narrow. We are going to extend these results to the so-called hereditary-SCD-operators.

Definition 5.10. Let $X$ and $Y$ be Banach spaces. A bounded linear operator $T: X \longrightarrow Y$ is said to be a hereditary-SCD-operator if every convex subset of $T\left(B_{X}\right)$ is an SCD set.

Here is the promised result.

Theorem 5.11. Let $X$ be a Banach space with Daugavet property and $T: X \longrightarrow Y$ be a hereditary-SCD-operator. Then, $T$ is narrow.

We need the following lemma, which could be of independent interest.

Lemma 5.12. Let $T: X \longrightarrow Y$ be a hereditary-SCD-operator. Then, for every $x^{*} \in X^{*}$ the operator $T \tilde{+} \operatorname{Re} x^{*}: X \longrightarrow Y \oplus_{1} \mathbb{R}$ is an SCD-operator.

Proof. Denote $P_{1}:\left[T \tilde{+} \operatorname{Re} x^{*}\right](X) \longrightarrow T(X)$ and $P_{2}:\left[T \tilde{+} \operatorname{Re} x^{*}\right](X) \longrightarrow \mathbb{R}$ as the natural coordinate projections. What we need to show is that there is a determining sequence of relatively weakly-open subsets of the set $A=\left[T \tilde{+} \operatorname{Re} x^{*}\right]\left(B_{X}\right)$. Since $A$ is separable, it is enough to prove that for every $a \in A$ there exists a sequence of relatively weakly open sets $\left\{V_{n}: n \in \mathbb{N}\right\}$ such that for every $B \subseteq A$ intersecting all the $V_{n}, a \in \overline{\operatorname{conv}}(B)$ (see Remark 2.4).

We fix $a \in A$ and denote

$$
A_{a}=\left\{b \in A: P_{1}(b)=P_{1}(a)\right\} .
$$

It is easy to see that $A_{a}$ is of the form

$$
A_{a}=\left\{\left(P_{1}(a), t\right): t \in \Delta_{a}\right\},
$$

where $\Delta_{a}$ is a bounded interval in $\mathbb{R}$. We denote $\alpha_{a}=\inf \Delta_{a}$ and $\beta_{a}=\sup \Delta_{a}$, and we consider

$$
S_{n, 1}=\left\{b \in A: P_{2}(b)<\alpha_{a}+\frac{1}{n}\right\} \quad \text { and } \quad S_{n, 2}=\left\{b \in A: P_{2}(b)>\beta_{a}-\frac{1}{n}\right\},
$$

which are nonempty slices of $A$ (since they intersect $A_{a}$ ) for all $n \in \mathbb{N}$ and $i=1,2$. Now, since $T$ is a hereditary-SCD-operator and $P_{1}\left(S_{n, i}\right) \subseteq T\left(B_{X}\right)$ is convex, for every $n \in \mathbb{N}$ and $i=1,2$ we may find a determining sequence $\left\{S_{n, i}^{m}: m \in \mathbb{N}\right\}$ of slices of $P_{1}\left(S_{n, i}\right)$. We write

$$
V_{n, i}^{m}=S_{n, i} \cap P_{1}^{-1}\left(S_{n, i}^{m}\right) \quad(n, m \in \mathbb{N}, i=1,2),
$$

which are relatively weakly open subsets of $A$. We will prove that they are the sets we need. Indeed, let $B \subseteq A$ be such that

$$
\emptyset \neq B \cap V_{n, i}^{m}=B \cap S_{n, i} \cap P_{1}^{-1}\left(S_{n, i}^{m}\right) \quad(n, m \in \mathbb{N}, i=1,2) .
$$

For every $n \in \mathbb{N}$, we observe that

$$
P_{1}\left(B \cap S_{n, i}\right) \cap S_{n, i}^{m} \neq \emptyset \quad(m \in \mathbb{N}, i=1,2),
$$


so, since the sequences $\left\{S_{n, i}^{m}: m \in \mathbb{N}\right\}$ are determining, we get that

$$
\overline{P_{1}\left(B \cap S_{n, i}\right)}=\overline{\operatorname{conv}}\left(P_{1}\left(B \cap S_{n, i}\right)\right) \supseteq P_{1}\left(S_{n, i}\right) \quad(i=1,2) .
$$

In particular, $\overline{P_{1}\left(B \cap S_{n, i}\right)} \ni P_{1}(a)$, meaning that for every $n \in \mathbb{N}$, every $i=1,2$, and every $\varepsilon>0$, there exists $x_{n, i}^{\varepsilon} \in B \cap S_{n, i}$ such that

$$
\left\|P_{1}\left(x_{n, i}^{\varepsilon}\right)-P_{1}(a)\right\| \leqslant \varepsilon .
$$

Now, we fix some $\varepsilon>0$ and, since obviously $a \in A_{a}$, we may take $n \in \mathbb{N}$ such that

$$
\alpha_{a}+\frac{1}{n}-\varepsilon<P_{2}(a)<\beta_{a}-\frac{1}{n}+\varepsilon .
$$

So, for the corresponding $x_{n, 1}^{\varepsilon}$ and $x_{n, 2}^{\varepsilon}$, we have

$$
P_{2}\left(x_{n, 1}^{\varepsilon}\right)-\varepsilon<P_{2}(a)<P_{2}\left(x_{n, 2}^{\varepsilon}\right)+\varepsilon .
$$

Then, there is a convex combination

$$
x_{n}^{\varepsilon}=\lambda_{1} x_{n, 1}^{\varepsilon}+\lambda_{2} x_{n, 2}^{\varepsilon} \quad\left(\lambda_{1}+\lambda_{2}=1\right)
$$

(so $\left.x_{n}^{\varepsilon} \in \operatorname{conv}(B)\right)$ such that

$$
\left|P_{2}\left(x_{n}^{\varepsilon}\right)-P_{2}(a)\right|<\varepsilon .
$$

This, together with (7), implies that $\left\|x_{n}^{\varepsilon}-a\right\|<2 \varepsilon$, and the arbitrariness of $\varepsilon>0$ gives us the fact that $a \in \overline{\operatorname{conv}}(B)$.

Proof of Theorem 5.11, To prove that $T$ is narrow, it is enough to show that for every $x^{*} \in X^{*}$, the operator $T \tilde{+} \operatorname{Re} x^{*}$ is a strong Daugavet operator. But this fact follows from Lemma 5.12 and Proposition 5.8

As we did for the alternative Daugavet property in Corollary [5.5, we can extend Theorem 5.11 to the nonseparable case.

Corollary 5.13. Let $X$ be a Banach space with the Daugavet property and let $T \in$ $L(X)$ be such that $\left.T\right|_{Y}$ is a hereditary-SCD-operator for every separable subspace $Y$ of $X$. Then, $T$ is narrow and, in particular, $\|\mathrm{Id}+T\|=1+\|T\|$.

Proof. We fix $x, y \in S_{X}$, a slice $S$ of $B_{X}$ and $\varepsilon>0$. We take a separable subspace $Y_{1}$ of $X$ such that $x, y \in Y_{1}$ and such that $S \cap Y_{1} \neq \emptyset$, and we follow the proof of Corollary [5.5, using [20, Theorem 4.5] instead of Remark [4.6, to get a separable subspace $Y$ of $X, T$-invariant, with the Daugavet property and such that $x, y \in S_{X}$ and $S \cap Y \neq \emptyset$. Now, $\left.T\right|_{Y}$ is a hereditary-SCD-operator, so Theorem 5.11 gives us the fact that $\left.T\right|_{Y}$ is narrow. Then, we may find $z \in S \cap Y \subseteq S$ such that $\|x+z\| \geqslant 2-\varepsilon$ and

$$
\|T y-T z\|=\left\|T_{Y}(y)-\left.T\right|_{Y}(z)\right\|<\varepsilon .
$$

The following particular cases are especially interesting. The first one was proved in [20, Theorem 4.13] with a different argument.

Corollary 5.14. Let $X$ be a Banach space with the Daugavet property and let $T \in L(X)$ be an operator which does not fix copies of $\ell_{1}$. Then, $T$ is narrow.

Corollary 5.15. Let $X$ be a Banach space with the Daugavet property and let $T \in L(X)$ be an operator such that $T\left(B_{X}\right)$ is strongly regular. Then, $T$ is narrow. 
Remarks 5.16.

(a) The class of hereditary-SCD-operators is a right operator ideal. Indeed, if $T: X_{1} \longrightarrow X_{2}$ is an arbitrary operator and $S: X_{2} \longrightarrow X_{3}$ is a hereditarySCD-operator, then $[S T]\left(B_{X_{1}}\right) \subseteq S\left(\|T\| B_{X_{2}}\right)$, so $S T$ is an hereditary-SCDoperator.

(b) The class of hereditary-SCD-operators is not a left operator ideal. Indeed, we consider a norm-one projection $T: L_{1}[0,1] \longrightarrow X \equiv \ell_{1}$ which is a hereditary-SCD-operator since $\ell_{1}$ is RNP. We also consider a quotient map $S: \ell_{1} \longrightarrow \ell_{1} / Y \equiv L_{1}[0,1]$ (by just using the factor universality of $\ell_{1}$ ). Then, $S T\left(B_{L_{1}[0,1]}\right)=B_{L_{1}[0,1]}$, so $S T$ is not even an SCD-operator.

(c) As a consequence, there are narrow operators which are not $S C D$-operators. Indeed, since the set of narrow operators is clearly a left operator ideal, the operator $S T$ above is narrow.

\section{Countable $\pi$-Bases of the weak topology}

It was shown in Proposition 2.21 that a convex bounded subset $A$ of a Banach space $X$ is SCD if it has a countable $\pi$-base of the weak topology. But we do not know whether these two properties are equivalent. The aim of this section is to discuss this possible equivalence. In a first subsection we will show that the class of sets having countable $\pi$-bases of the weak topology contains separable CPCP sets. We already know that it contains those sets which do not have $\ell_{1}$-sequences (Theorem 2.22), so this class covers most of the examples of SCD sets presented in this paper. In the second subsection we will show that convex bounded subsets of both $\ell_{1}\left(c_{0}\right)$ and $c_{0}\left(\ell_{1}\right)$ also have countable $\pi$-bases of the weak topology. Finally, the third subsection contains several characterizations of SCD sets which remind us of the property we are dealing with.

6.1. CPCP sets. We start with a sufficient condition to have a countable $\pi$-base of the weak topology.

Proposition 6.1. Let $X$ be a Banach space and let $A$ be a separable closed convex bounded subset of $X$ such that there is a weakly dense subset $B$ of $A$ consisting of points of continuity of Id $:\left(A, \sigma\left(X, X^{*}\right)\right) \longrightarrow(A,\|\cdot\|)$. Then, $\left(A, \sigma\left(X, X^{*}\right)\right)$ has a countable $\pi$-base.

Proof. Let $D$ be a countable norm dense subset of $B$, and for every $d \in D$ and every $n \in \mathbb{N}$ let $U_{d}^{n}$ be a weak open neighborhood of $d$ in $A$ of diameter less than $\frac{1}{n}$. We claim that the countable family $\left\{U_{d}^{n}: n \in \mathbb{N}, d \in D\right\}$ is a $\pi$-base of $A$. Indeed, let $W$ be a weakly open subset of $A$. Since $B$ is weakly dense in $A, W \cap B$ is nonempty and relatively norm open in $B$, so, since $D$ is norm dense in $B$, there is $d \in D \cap W$. Now, $W$ is a norm open neighborhood of $d$ relative to $A$, so it contains $B(d, 1 / n) \cap A$ for some $n \in \mathbb{N}$, and so $U_{d}^{n} \subseteq W$. We are done.

A first consequence of the above result deals with LUR renorming. It is clear from the definition that denting points are points of weak-norm continuity of the identity map, and so, as it was commented before Example 2.10, the unit ball of a Banach space with an LUR norm fulfills the above condition. It was also commented there that every separable Banach space can be equivalently renormed with an LUR norm. 


\section{Example 6.2.}

(a) Let $X$ be a separable Banach space with an LUR norm. Then, $B_{X}$ has a countable $\pi$-base of the weak topology.

(b) As a consequence, every separable Banach space $X$ admits an equivalent norm $|\cdot|$ such that $B_{(X,|\cdot|)}$ has a countable $\pi$-base of the weak topology.

We are going to show that $\mathrm{CPCP}$ sets have countable $\pi$-bases for the weak topology. We recall that a closed convex bounded subset $A$ of a Banach space $X$ has the $\mathrm{CPCP}$ if every closed convex subset $B$ of $A$ contains a weak-to-norm point of continuity of the identity mapping. In this case, for every convex subset $B$ of $A$ and for every $\varepsilon>0$, there is a relatively weakly open subset $C \subseteq B$ with $\operatorname{diam}(C)<\varepsilon$ [5]. We need the following result which follows from [14, Lemma I.0]; we have not found a direct reference, so we include a proof for the sake of completeness.

Lemma 6.3. Let $X$ be a Banach space and let $A$ be a closed convex bounded subset of $X$ with the CPCP. Then, there is a weakly dense subset $D$ of $A$ consisting of points of weak-norm continuity of $\operatorname{Id}:\left(A, \sigma\left(X, X^{*}\right)\right) \longrightarrow(A,\|\cdot\|)$.

Proof. We fix a sequence of positive $\varepsilon_{n}$ tending to zero and write

$$
D_{n}=\bigcup\left\{C: C \text { is weakly open in } A \text { and } \operatorname{diam}(C)<\varepsilon_{n}\right\} .
$$

Let us prove that $D=\bigcap_{n \in \mathbb{N}} D_{n}$ is weakly dense in $A$. Indeed, let $U \subseteq A$ be relatively weakly open. We pick $U_{1} \subseteq U$ closed convex with nonempty interior. Then, there is a relatively weakly open subset $C_{1}$ of $A$ of diameter less than $\varepsilon_{1}$ such that $C_{1}$ is contained in the weak interior of $U_{1}$. We repeat the process to find a decreasing sequence $C_{n}$ of weakly open sets with nonempty interior such that $\operatorname{diam}\left(C_{n}\right)<\varepsilon_{n}$ and $\overline{C_{n+1}} \subseteq C_{n}$. Then, the Cantor theorem tells us that there is $x \in \bigcap_{n \in \mathbb{N}} \overline{C_{n}}$. Now, we have in particular that $x \in C_{1} \subseteq U_{1} \subseteq U$. On the other hand, for every $n \in \mathbb{N}, x \in C_{n}$ and $\operatorname{diam}\left(C_{n}\right)<\varepsilon_{n}$, then $x \in D_{n}$. Therefore, $x \in D$. Finally, every point of $D$ has weak neighborhoods of arbitrarily small diameter, showing that it is a point of continuity.

This result, together with Proposition 6.1 gives the main result of the subsection.

Corollary 6.4. Let $X$ be a Banach space and let $A$ be a separable closed convex bounded subset of $X$ with the CPCP. Then, $A$ has a countable $\pi$-base for the weak topology.

With the above result, most of the types of SCD sets presented in section 2 have a countable $\pi$-base of the weak topology. The only exception is the family of strongly regular sets which are not CPCP. There are two main examples of sets of this kind, but in both cases, the sets have a countable $\pi$-base of the weak topology.

\section{Examples 6.5.}

(a) The set constructed by S. Argyros, E. Odell, and H. Rosenthal [2] which is strongly regular but does not have the CPCP is a subset of $c_{0}$, so it has a countable $\pi$-base of the weak topology since it does not have $\ell_{1}$-sequences.

(b) The set constructed by W. Schachermayer [28] is a subset $C$ of a Banach space $Z$ which does not have the CPCP but where $Z^{* *}$ is strongly regular (so $Z$ is strongly regular). But then, $\left(C, \sigma\left(X, X^{*}\right)\right)$ has a countable $\pi$-base of the weak topology since $Z$ does not contain $\ell_{1}$. 
6.2. $c_{0}\left(\ell_{1}\right)$ and $\ell_{1}\left(c_{0}\right)$. Our goal in this subsection is to show that convex bounded subsets of the spaces $c_{0}\left(\ell_{1}\right)$ and $\ell_{1}\left(c_{0}\right)$ have a countable $\pi$-base of the weak topology. The first case is easier to demonstrate.

Example 6.6. Every convex bounded subset $A$ of the space $c_{0}\left(\ell_{1}\right)$ has a countable $\pi$-base of the weak topology.

Proof. Let $X$ denote $c_{0}\left(\ell_{1}\right)$. For every $m \in \mathbb{N}$, we denote

$$
Y_{m}=\left[\ell_{1} \oplus \ell_{1} \oplus \cdots \cdot \cdots \oplus \ell_{1} \oplus 0 \oplus 0 \oplus \cdots\right]_{\infty} \subseteq c_{0}\left(\ell_{1}\right)
$$

and $P_{m}: X \longrightarrow Y_{m}$ for the natural projection. Since $P_{m}(A)$ is a convex bounded subset of $Y_{m}$ and $Y_{m}$ is isomorphic to $\ell_{1}$, there is a countable $\pi$-base $\left\{S_{m, k}: k \in \mathbb{N}\right\}$ of $\left(P_{m}(A), \sigma\left(Y_{m}, Y_{m}^{*}\right)\right)$. We are going to prove that the collection

$$
\widetilde{S}_{m, k}=\left[P_{m}^{-1}\left(S_{m, k}\right)\right] \cap A \quad(m, k \in \mathbb{N})
$$

forms a countable $\pi$-base of $\left(A, \sigma\left(X, X^{*}\right)\right)$. Indeed, let $U, V$ be weak neighborhoods of $0, V+V \subseteq U, a \in A$, and denote $B=(a+U) \cap A$. Every relatively weakly open subset of $A$ is of the same form as $B$, so we have to prove that $\widetilde{S}_{m, k} \subseteq B$ for some choice of $m$ and $k$. Assume to the contrary that none of $\widetilde{S}_{m, k}$ is contained in $B$. For $m \in \mathbb{N}$ large enough, all the $P_{m}(A)$ intersect $(a+V)$. Fix $m \in \mathbb{N}$ with $C_{m}=$ $(a+V) \cap P_{m}(A) \neq \emptyset$. Then there is $k(m) \in \mathbb{N}$ with $S_{m, k(m)} \subseteq C_{m}$. According to our assumption $\widetilde{S}_{m, k(m)}$ is not contained in $B$, so there is an $x_{m} \in \widetilde{S}_{m, k(m)} \backslash B$. This $x_{m}$ can be written as $x_{m}=y_{m}+z_{m}$, where $y_{m} \in S_{m, k(m)} \subseteq C_{m}$ and $z_{m} \in \operatorname{Ker} P_{m}$. Since $x_{m} \in A$ and $y_{m} \in P_{m}(A)$, we have the fact that $z_{m}$ is a bounded sequence, and since by our construction $\left(z_{m}\right)$ tend to 0 coordinate-wise as $m \rightarrow \infty$, we can deduce that $\left(z_{m}\right) \longrightarrow 0$ in the weak topology. Therefore, for some $m$ large enough $z_{m} \in V$, and consequently $x_{m}=y_{m}+z_{m} \in(a+V)+V \subseteq a+U$. Since $x_{m} \in A$, this means that $x_{m} \in(a+U) \cap A=B$, which contradicts the selection of $x_{m}$.

Remark 6.7. The argument above also works for $c_{0}$-sums of RNP spaces. Indeed, this follows from the fact that a finite-sum of RNP spaces is again an RNP space (see [9, Theorem 6.5.b], for instance).

Let us remark with an example that to have a countable $\pi$-base of the weak topology does not imply that any point has a countable base of weak neighborhoods.

Example 6.8. The unit ball of $X=c_{0}\left(\ell_{1}\right)$ has no point with a countable base of relative weak neighborhoods. Indeed, we consider an arbitrary $x=\left(x_{n}\right)_{n \in \mathbb{N}} \in B_{X}$, where $x_{n} \in \ell_{1},\left\|x_{n}\right\| \longrightarrow 0$ and $\max _{n \in \mathbb{N}}\left\|x_{n}\right\| \leqslant 1$. We fix $n_{0} \in \mathbb{N}$ such that $\left\|x_{n_{0}}\right\|<1 / 2$, and we consider the subset

$$
A=\left\{\left(y_{n}\right)_{n \in \mathbb{N}} \in B_{X}: y_{n}=x_{n} \text { if } n \neq n_{0},\left\|x_{n_{0}}-y_{n_{0}}\right\| \leqslant 1 / 2\right\} .
$$

Then, $A$ is a closed subset of $B_{X}$ containing $x$, so if $x$ has a countable base of relative weak neighborhoods in $B_{X}$, then $x$ also has a countable base of relative weak neighborhoods in $A$. But the latter is impossible, because $A$ is affinely homeomorphic to $B_{\ell_{1}}$, with $x$ being the image of $0 \in B_{\ell_{1}}$.

To get the second example we need a technical result. 
Lemma 6.9. Let $X$ be a separable Banach space. Then, the following are equivalent:

(i) Every convex bounded subset of $X$ has a countable $\pi$-base of the weak topology.

(ii) Every closed convex bounded subset $A$ of $X$ has a point with a countable local $\pi$-base of relatively weakly open subsets (i.e. there is $x \in A$ and a sequence $\left\{U_{n}: n \in \mathbb{N}\right\}$ of relatively weakly open subsets of $A$ such that for every relative weak neighborhood $V$ of $x$ there is some $U_{n} \subseteq V$ ).

(iii) For every $\varepsilon>0$, every closed convex bounded subset $A$ of $X$ has a point with a countable local $\varepsilon$-base of relatively weakly open subsets (i.e. there is $x \in A$ and a sequence $\left\{U_{n}: n \in \mathbb{N}\right\}$ of relatively weakly open subsets of $A$ such that for every weakly open neighborhood $V \subseteq X$ of $x$ in the whole space there is $n \in \mathbb{N}$ with $\left.U_{n} \subseteq V+\varepsilon B_{X}\right)$.

Proof. (i) $\Rightarrow$ (ii) $\Rightarrow$ (iii) are clear since a $\pi$-base is a local $\pi$-base, and a local $\pi$-base is an $\varepsilon$-base for every $\varepsilon>0$.

(iii) $\Rightarrow$ (i). It is straightforward to show that it is enough to deal with closed convex bounded subsets of $X$. Just observe that if $\left\{U_{n}: n \in \mathbb{N}\right\}$ is a $\pi$-base for the weak topology of the closure of a convex bounded subset $A$ of $X$, then $\left\{U_{n} \cap A: n \in \mathbb{N}\right\}$ is a $\pi$-base of the weak topology of $A$ itself.

We then fix a closed convex bounded subset $A \subseteq X$. We first remark that, for every $\varepsilon>0$, the subset $B_{\varepsilon} \subseteq A$ of points having a countable local $\varepsilon$-base is weakly dense in $A$. Indeed, we consider an arbitrary weakly open subset $U$ of $X$ intersecting $A$, and we fix another weakly open subset $V \subseteq U \subseteq X$ intersecting $A$ with $\bar{V}^{\sigma\left(X, X^{*}\right)} \subseteq U$. According to our assumption, there is $x \in \bar{V}^{\sigma\left(X, X^{*}\right)} \cap A$ with a local $\varepsilon$-base $\left\{U_{n}: n \in \mathbb{N}\right\}$. But then, $V_{n}=U_{n} \cap V$ form a countable local $\varepsilon$-base of relatively weakly open subsets of $A$ for $x$, i.e. $x \in B_{\varepsilon} \cap U$, so $B \cap U \neq \emptyset$.

Now, for every $k \in \mathbb{N}$ we take a countable norm dense subset $\left\{b_{k, m}: m \in \mathbb{N}\right\}$ in $B_{1 / k}$, and for every $b_{k, m}$ we select a $1 / k$-base $\left\{U_{k, m, n}: n \in \mathbb{N}\right\}$. Let us show that $\left\{U_{k, m, n}: k, n, m \in \mathbb{N}\right\}$ forms a $\pi$-base for $\left(A, \sigma\left(X, X^{*}\right)\right)$. Indeed, let $U, V$ be weak neighborhoods of $0, V+V \subseteq U, a \in A$, and denote $G=(a+U) \cap A$. We have to prove that $U_{k, m, n} \subseteq G$ for some choice of $k, m, n \in \mathbb{N}$. To do this, we take $k \in \mathbb{N}$ large enough that $\frac{1}{k} B_{X} \subseteq V$. According to our construction, there is $m \in \mathbb{N}$ with $b_{k, m} \in(a+V) \cap A$. Then, there is $n \in \mathbb{N}$ with $U_{k, m, n} \subseteq(a+V)+\frac{1}{k} B_{X}$. Therefore

$$
U_{k, m, n} \subseteq\left(a+V+\frac{1}{k} B_{X}\right) \cap A \subseteq(a+V+V) \cap A \subseteq(a+U) \cap A=G .
$$

We are now able to present the second example.

Example 6.10. Every convex bounded subset $A$ of the space $\ell_{1}\left(c_{0}\right)$ has a countable $\pi$-base of the weak topology.

Proof. Let $X$ denote $\ell_{1}\left(c_{0}\right)$. For $\varepsilon>0$ fixed, arguing the same way as in the beginning of the proof of Theorem 3.12, we select an open slice $S \subseteq A$ and an $m \in \mathbb{N}$ with the following property:

$$
\left\|\left(0, \ldots, 0, y_{m+1}, y_{m+2}, \ldots\right)\right\|<\frac{\varepsilon}{2} \quad\left(\left(y_{n}\right)_{n \in \mathbb{N}} \in S\right) .
$$

Let us prove that every $x_{0} \in S$ has a countable $\varepsilon$-base of relatively weakly open subsets, and Lemma 6.9 will give the result. 
We denote

$$
Y_{m}=\left[c_{0} \oplus c_{0} \oplus \stackrel{m}{*} \oplus c_{0} \oplus 0 \oplus 0 \oplus \cdots\right]_{\ell_{1}} \subseteq \ell_{1}\left(c_{0}\right)
$$

and let $P_{m}: X \longrightarrow Y_{m}$ be the natural projection. Since $Y_{m}$ is isomorphic to $c_{0}$, there is a countable local $\pi$-base $\left\{U_{n}: n \in \mathbb{N}\right\}$ of $P_{m}\left(x_{0}\right)$ in $\left(P_{m}(S), \sigma\left(X, X^{*}\right)\right)$. Consider

$$
\widetilde{U_{n}}=P_{m}^{-1}\left(U_{n}\right) \cap S \quad(n \in \mathbb{N}),
$$

which are weakly open subsets of $S$, and hence they are weakly open in $A$. Let us show that $\left\{\widetilde{U_{n}}: n \in \mathbb{N}\right\}$ forms an $\varepsilon$-base for $x_{0}$ in $A$. Consider a weakly open neighborhood $V \subseteq X$ of $x_{0}$. By (8), we have that

$$
\left\|P_{m}(y)-y\right\|<\varepsilon / 2 \quad(y \in S) .
$$

So $\left(V+\frac{\varepsilon}{2} B_{X}\right) \cap P_{m}(S)$ is a weak neighborhood of $P_{m}\left(x_{0}\right)$ in $P_{m}(S)$. So there is an $n \in \mathbb{N}$ such that $U_{n} \subseteq V+\frac{\varepsilon}{2} B_{X}$. Applying (9) once more, we obtain that

$$
\widetilde{U_{n}}=P_{m}^{-1}\left(U_{n}\right) \cap S \subseteq U_{n}+\frac{\varepsilon}{2} B_{X} \subseteq V+\varepsilon B_{X} .
$$

6.3. Two characterizations of SCD sets. The aim of this part of the section is to establish some characterizations of SCD sets which remind us of countable $\pi$-bases of the weak topology. The first one deals with convex combinations of slices.

Theorem 6.11. A convex bounded subset $A$ of a Banach space $X$ is an SCD set if and only if there is a sequence $\left\{V_{n}: n \in \mathbb{N}\right\}$ of convex combinations of slices of A such that every relatively weakly open subset of A contains some of the $V_{n}$.

Proof. The "if" part is direct consequence of Propositions 2.2 and 2.18 .

Conversely, assume that $A$ is an SCD set and suppose without loss of generality that $A \subseteq B_{X}$. Let $S_{n}=S\left(A, x_{n}^{*}, \varepsilon_{n}\right)$, for $n \in \mathbb{N}$, be a determining sequence of slices for $A$. Let us show that the convex combinations of the $S_{n}$ 's with rational coefficients form the countable collection of convex combinations of slices that we need. Indeed, let $U$ be a relatively weakly open subset of $A$. Select another relatively weakly open subset $V \subseteq U$ such that $\alpha=\operatorname{dist}(V, A \backslash U)>0$. Due to Bourgain's lemma (Lemma 2.16), there is a convex combination of slices $\sum_{j=1}^{m} \lambda_{j} G_{j} \subseteq V$. According to Proposition 2.2, for every $j=1,2, \ldots, m$ there is $n(j) \in \mathbb{N}$ such that $S_{n(j)} \subseteq G_{j}$. Then, $\sum_{j=1}^{m} \lambda_{j} S_{n(j)} \subseteq V$. What remains is to find rationals $\mu_{j}>0$ with $\sum_{j=1}^{m} \mu_{j}=1$ and $\left|\mu_{j}-\lambda_{j}\right|<\alpha$. Then, the Hausdorff distance between $\sum_{U}^{m}{ }_{j=1}^{m} \mu_{j} S_{n(j)}$ and $\sum_{j=1}^{m} \lambda_{j} S_{n(j)}$ is smaller than $\alpha$, so $\sum_{j=1}^{m} \mu_{j} S_{n(j)} \subseteq V+\alpha B_{X} \subseteq$

The second result gives a reformulation of SCD in terms of topological properties of the set of extreme points of its weak* closure in the bidual. For a convex bounded subset $A$ of a Banach space $X$, denote $\bar{A}^{* *}$ as the weak-star closure of $A$ in $X^{* *}$.

Theorem 6.12. Let $X$ be a Banach space and let $A$ be a convex bounded subset of $X$. Put $W=\left(\operatorname{ext}\left(\bar{A}^{* *}\right), \sigma\left(X^{* *}, X^{*}\right)\right)$. Then, the following are equivalent:

(i) $A$ is an $S C D$ set.

(ii) $W$ has a countable $\pi$-base. 
Proof. (i) $\Longrightarrow$ (ii). We take a sequence of slices $S_{n}=S\left(A, x_{n}^{*}, \varepsilon_{n}\right)$ for $n \in \mathbb{N}$ which is determining for $A$, and we write

$$
S_{n}^{* *}=S\left(\bar{A}^{* *}, x_{n}^{*}, \varepsilon_{n}\right) \subseteq \bar{A}^{* *}
$$

for the natural extensions of $S_{n}$ to slices of $\bar{A}^{* *}$. Then, the family $U_{n}=S_{n}^{* *} \cap W$ for $n \in \mathbb{N}$ forms a $\pi$-base of $W$. Indeed, we consider a relatively weak*-open subset $U$ of $W$. Due to Choquet's lemma (i.e., for any locally convex topology, slices containing an extreme point of a compact convex set make up a neighborhood base of the extreme point; see [10, Definition 25.3 and Proposition 25.13]), there is a slice $S^{* *}=S\left(\bar{A}^{* *}, x^{*}, \varepsilon\right)$ of $\bar{A}^{* *}$ generated by some $x^{*} \in X^{*}$ and $\varepsilon>0$ such that $U \supseteq S^{* *} \cap W \neq \emptyset$. Now, according to Proposition 2.2, there is an $n \in \mathbb{N}$ such that

$$
S_{n} \subseteq S\left(A, x^{*}, \varepsilon / 2\right) \subseteq S\left(\bar{A}^{* *}, x^{*}, \varepsilon / 2\right) .
$$

Then, $S_{n}^{* *}$ is contained in the relative weak ${ }^{*}$-closure of $S\left(\bar{A}^{* *}, x^{*}, \varepsilon / 2\right)$ in $\bar{A}^{* *}$, so $S_{n}^{* *} \subseteq S^{* *}$ and

$$
U_{n}=S_{n}^{* *} \cap W \subseteq S^{* *} \cap W \subseteq U .
$$

(ii) $\Longrightarrow$ (i). We consider a countable $\pi$-base $\left\{U_{n}: n \in \mathbb{N}\right\}$ of $W$ consisting of relatively weak*-star open subsets. Again by Choquet's lemma, there are $x_{n}^{*} \in X^{*}$ and $\varepsilon_{n}>0$ such that

$$
U_{n} \supseteq \widetilde{U_{n}}=S\left(\bar{A}^{* *}, x_{n}^{*}, \varepsilon_{n}\right) \cap W \neq \emptyset .
$$

Let us prove that the slices $S_{n, m}=S\left(A, x_{n}^{*}, 1 / m\right)$ with $n, m \in \mathbb{N}$ form a determining sequence for $A$. Indeed, we denote $S_{n, m}^{* *}$ as the closed slices of $\bar{A}^{* *}$ generated by $x_{n}^{*}$ and $1 / m$. For every slice $S=S\left(A, x^{*}, \varepsilon\right)$ of $A$, since $\left\{\widetilde{U_{n}}: n \in \mathbb{N}\right\}$ is a $\pi$-base of $W$, there is $n \in \mathbb{N}$ such that

$$
S^{* *} \cap W \supseteq \widetilde{U_{n}}, \quad \text { where } S^{* *}=S\left(\bar{A}^{* *}, x^{*}, \varepsilon_{n}\right),
$$

so for $m \in \mathbb{N}$ large enough we have

$$
S^{* *} \cap W \supseteq S_{n, m}^{* *} \cap W .
$$

Then, taking into account that, for every $n \in \mathbb{N}$,

$$
G_{n}=\bigcap_{m \in \mathbb{N}} S_{n, m}^{* *}
$$

is a closed face of $\bar{A}^{* *}$, the Krein-Milman theorem gives us the fact that

$$
G_{n}=\overline{\operatorname{conv}\left(G_{n} \cap W\right)}{ }^{\sigma\left(X^{* *}, X^{*}\right)} .
$$

Therefore,

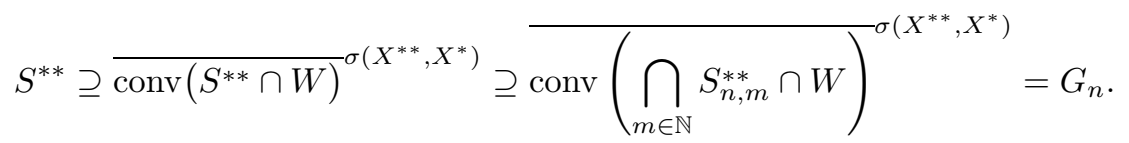

This means that the intersection of the decreasing sequence of $\sigma\left(X^{* *}, X^{*}\right)$ compact sets $\left\{S_{n, m}^{* *}: m \in \mathbb{N}\right\}$ is contained in $S^{* *}$. But $S^{* *}$ is a relatively $\sigma\left(X^{* *}, X^{*}\right)$ open set in $\bar{A}^{* *}$, so for sufficiently large $m \in \mathbb{N}$, all the $S_{n, m}^{* *}$ are subsets of $S^{* *}$. For these $m$, we have

$$
S=S^{* *} \cap A \supseteq S_{n, m}^{* *} \cap A \supseteq S_{n, m} .
$$

Finally, we use the characterization of SCD sets from Proposition 2.2 . 
The following is an easy consequence of the above result.

Corollary 6.13. Let $X$ be a Banach space and let $A$ be a convex bounded subset of $X$. If $A$ is $S C D$, then $\left(\operatorname{ext}\left(\bar{A}^{* *}\right), \sigma\left(X^{* *}, X^{*}\right)\right)$ is separable.

The particular case of the above corollary for subsets of separable Banach spaces without copies of $\ell_{1}$ should be previously known. Anyway, we include an easy direct proof of this fact.

Remark 6.14. Let $X$ be a separable Banach space without copies of $\ell_{1}$ and let $A$ be a convex bounded subset of $X$. Then, $\left(\operatorname{ext}\left(\bar{A}^{* *}\right), \sigma\left(X^{* *}, X^{*}\right)\right)$ is separable. Indeed, we write

$$
C=\operatorname{conv}\left(\operatorname{ext}\left(\bar{A}^{* *}\right)\right)
$$

and we observe that $C$ is $\sigma\left(X^{* *}, X^{*}\right)$-sequentially dense in its weak*-closure $\bar{A}^{* *}$ (see [12, Theorem 4.1]). Then, we take a sequence $\left\{y_{n}: n \in \mathbb{N}\right\}$ dense in $A$ and consider those extreme points of $\bar{A}^{* *}$ needed to approximate each $y_{n}$ by a sequence of convex combinations. The union of all these extreme points (while countable) is weak*-dense in the set of all extreme points of $\bar{A}^{* *}$ by the reversed Krein-Milman theorem.

\section{Open qUESTIONS}

Question 7.1. Let $X$ be a Banach space and let $A$ be a convex bounded subset of $X$. If $A$ is SCD, does $A$ have a countable $\pi$-base for the weak topology?

Question 7.2. Let $X$ be an SCD space. Does every convex bounded subset of $X$ have a countable $\pi$-base for the weak topology?

Related to these questions is the following one.

Question 7.3. Let $L$ be a compact subset of a locally convex space and let $K$ be its closed convex hull. If $L$ has a countable $\pi$-base, does it imply that $K$ also has a countable $\pi$-base? What if $L=\operatorname{ext}(K)$ ?

Let us explain why this question is related to the above two. Observe that if $D$ is a dense subspace of a topological space $E$ and $\mathcal{B}$ is a $\pi$-base for $E$, then $\{B \cap D: B \in \mathcal{B}\}$ is a $\pi$-base for $D$. In particular, if $\left(\bar{A}^{* *}, \sigma\left(X^{* *}, X^{*}\right)\right)$ has a countable $\pi$-base, then so does $\left(A, \sigma\left(X, X^{*}\right)\right)$. Thus, a positive answer to the preceding question combined with Theorem 6.12 would imply a positive answer to Questions 7.1 and 7.2 .

\section{Questions 7.4.}

(a) Is every Banach space with unconditional basis SCD?

(b) A simpler case: let $X$ be a Banach space with 1-symmetric basis. Is $B_{X}$ an SCD set?

Question 7.5. Are the concepts of SCD sets and almost-SCD sets equivalent (see Remark 4.5 for the definition)?

Questions 7.6. Let $X$ be a separable Banach space such that no subspace of it can be renormed with the Daugavet property. Is $X$ SCD?

\section{Questions 7.7.}

(a) Is the sum of two SCD-operators an SCD-operator?

(b) Is the sum of two hereditary-SCD-operators a hereditary-SCD-operator? 


\section{ACKNOWLEDGMENTS}

We would like to thank Bill Johnson and Rafael Payá for useful comments on the subject of the paper. We also thank the referee for the careful reading of the manuscript and for multiple stylistic remarks.

\section{Note ADDED IN PROOF}

Questions 7.7 have recently been answered in the negative [Vladimir Kadets, Varvara Shepelska, Sums of SCD sets and their applications to SCD operators and narrow operators, Cent. Eur. J. Math. 8(1) (2010), 129-134. DOI: 10.2478/s11533009-0075-7].

\section{REFERENCES}

1. F. Albiac and N. J. Kalton, Topics in Banach space theory, Graduate Texts in Mathematics 233, Springer-Verlag, New York, 2006. MR2192298 (2006h:46005)

2. S. Argyros, E. Odell, and H. Rosenthal, On certain convex subsets of $c_{0}$, Functional analysis (Austin, TX, 1986-87), 80-111, Lecture Notes in Math., 1332, Springer, Berlin, 1988. MR967090 (89k:46030)

3. Y. Benyamini And J. Lindenstrauss, Geometric nonlinear functional analysis, vol. 1, American Mathematical Society Colloquium Publications 48, AMS, Providence, RI, 2000. MR.1727673 (2001b:46001)

4. J. Bourgain, La propriété de Radon-Nikodym, Publ. Math. de l'Univ. Pierre et Marie Curie 36, 1979.

5. J. Bourgain, Dentability and finite-dimensional decompositions, Studia Math.67 (1980), 135-148. MR583294 (81m:46034)

6. R. R. Bourgin, Geometric Aspects of Convex Sets with the Radon-Nikodym Property, Lecture Notes in Math. 993, Springer-Verlag, Berlin 1983. MR704815 (85d:46023)

7. K. Boyko, V. Kadets, M. Martín, And J. Merí, Properties of lush spaces and applications to Banach spaces with numerical index 1, Studia Math. 190 (2009), 117-133. MR.2461290

8. K. Boyko, V. Kadets, M. Martín, and D. Werner, Numerical index of Banach spaces and duality, Math. Proc. Cambridge Phil. Soc. 142 (2007), 93-102. MR2296393

9. J. M. F. Castillo, And M. Gonzalez, Three-space problem in Banach space theory, Lecture Notes in Mathematics 1667, Springer-Verlag, Berlin 1997. MR.1482801 (99a:46034)

10. G. Choquet, Lectures on Analysis. Volume II: Representation Theory, W. A. Benjamin, Inc., London, 1969. MR0250012 (40:3253)

11. R. Deville, G. Godefroy And V. Zizler, Smoothness and renormings in Banach spaces, Pitman Monographs and Surveys in Pure and Applied Mathematics 64, Longman Scientific \& Tecnical, London, 1993. MR.1211634 (94d:46012)

12. D. VAN Dulst, Characterizations of Banach spaces not containig $\ell_{1}$, CWI Tract 59, Stichting Mathematisch Centrum, Centrum voor Wiskunde en Informatica, Amsterdam, 1989. MR.1002733 (90h:46037)

13. J. Duncan, C. McGregor, J. Pryce, and A. White, The numerical index of a normed space, J. London Math. Soc. 2 (1970), 481-488. MR0264371(41:8967)

14. N. Ghoussoub, G. Godefroy, B. Maurey, and W. Schachermayer, Some topological and geometrical structures in Banach spaces, Memoirs of the AMS, Providence, RI, 1987. MR 912637 (89h:46024)

15. Y. Ivaknho, V. Kadets, and D. Werner, The Daugavet property for spaces of Lipschitz functions, Math. Scand. 101 (2007), 261-279. MR2379289(2009c:46014)

16. V. Kadets, M. Martín, J. Merí, and R. PayÁ, Convexity and smoothness of Banach spaces with numerical index one, Illinois J. Math. 53 (2009), 163-182.

17. V. Kadets, M. Martín, J. Merí, and V. Shepelska, Lushness, numerical index one and duality, J. Math. Anal. Appl. 357 (2009), no. 1, 15-24. MR2526802

18. V. Kadets. M. Martín, and R. PAyÁ, Recent progress and open questions on the numerical index of Banach spaces, Rev. R. Acad. Cien. Serie A. Mat. 100 (2006), 155-182. MR2267407 (2007h:46011) 
19. V. M. Kadets, R. V. Shvidkoy, G. G. Sirotkin, and D. Werner, Banach spaces with the Daugavet property, Trans. Amer. Math. Soc. 352 (2000), 855-873. MR.1621757(2000c:46023)

20. V. M. Kadets, R. V. Shvidkoy, and D. Werner, Narrow operators and rich subspaces of Banach spaces with the Daugavet property, Studia Math. 147 (2001), 269-298. MR.1853772 (2002f:46018)

21. V. Kadets And D. Werner, A Banach space with the Schur and the Daugavet property, Proc. Amer. Math. Soc. 132 (2004), 1765-1773. MR2051139 (2005b:46021)

22. J. Lindenstrauss and L. Tzafriri, Classical Banach Spaces. I, Springer-Verlag, Berlin, 1977. MR0500056 (58:17766)

23. G. López, M. Martín, and R. PAyÁ, Real Banach spaces with numerical index 1, Bull. London Math. Soc. 31 (1999), 207-212. MR1664125 (99k:46024)

24. M. Martín, The alternative Daugavet property for $C^{*}$-algebras and $J B^{*}$-triples, Math. Nachr. 281 (2008), 376-385. MR2392119

25. M. Martín and T. Oikhberg, An alternative Daugavet property, J. Math. Anal. Appl. 294 (2004), 158-180. MR2059797 (2005b:46023)

26. H. P. Rosenthal, On the structure of nondentable closed bounded convex sets, Adv. in Math. 70 (1988), 1-58. MR947756 (89g:46041)

27. R. A. RYAN, Introduction to Tensor Products of Banach spaces, Springer-Verlag, London, 2002. MR 1888309 (2003f:46030)

28. W. Schachermayer, An example concerning strong regularity and points of continuity in Banach spaces. Functional analysis (Austin, TX, 1986-87), 64-79, Lecture Notes in Math., 1332, Springer, Berlin, 1988. MR967089 (90a:46039)

29. R. V. Shvidkoy, Geometric aspects of the Daugavet property, J. Funct. Anal. 176 (2000), 198-212. MR.1784413 (2001h:46019)

30. S. TodorČEvić, Compact subsets of the first Baire class, J. Amer. Math. Soc. 12 (1999), 1179-1212. MR1685782 (2000d:54028)

31. L. WEIS, On the surjective (injective) envelope of strictly (co-) singular operators, Studia Math. 54 (1976), 285-290. MR0399908 (53:3749)

Departamento de Matemáticas, Universidad de Murcia, 30100 Murcia, Spain

E-mail address: avileslo@um.es

Department of Mechanics and Mathematics, Kharkov National University, Pl. SvoBODY 4, 61077 Kharkov, Ukraine

E-mail address: vova1kadets@yahoo.com

Departamento de Análisis Matemático, Facultad de Ciencias, Universidad de Granada, 18071 Granada, Spain

E-mail address: mmartins@ugr.es

Departamento de Análisis Matemático, Facultad de Ciencias, Universidad de Granada, 18071 Granada, Spain

E-mail address: jmeri@ugr.es

Department of Mechanics and Mathematics, Kharkov National University, Pl. SvoBODY 4, 61077 KHARKOV, UKRAINE

E-mail address: shepelskaya@yahoo.com 